\title{
Relationship Between Generalization and Diversity in Coevolutionary Learning
}

\author{
Siang Yew Chong, Member, IEEE, Peter Tiňo, and Xin Yao, Fellow, IEEE
}

\begin{abstract}
Games have long played an important role in the development and understanding of coevolutionary learning systems. In particular, the search process in coevolutionary learning is guided by strategic interactions between solutions in the population, which can be naturally framed as game playing. We study two important issues in coevolutionary learning-generalization performance and diversity-using games. The first one is concerned with the coevolutionary learning of strategies with high generalization performance, that is, strategies that can outperform against a large number of test strategies (opponents) that may not have been seen during coevolution. The second one is concerned with diversity levels in the population that may lead to the search of strategies with poor generalization performance. It is not known if there is a relationship between generalization and diversity in coevolutionary learning. This paper investigates whether there is such a relationship in coevolutionary learning through a detailed empirical study. We systematically investigate the impact of various diversity maintenance approaches on the generalization performance of coevolutionary learning quantitatively using case studies. The problem of the iterated prisoner's dilemma (IPD) game is considered. Unlike past studies, we can measure both the generalization performance and the diversity level of the population of evolved strategies. Results from our case studies show that the introduction and maintenance of diversity do not necessarily lead to the coevolutionary learning of strategies with high generalization performance. However, if individual strategies can be combined (e.g., using a gating mechanism), there is the potential of exploiting diversity in coevolutionary learning to improve generalization performance. Specifically, when the introduction and maintenance of diversity lead to a speciated population during coevolution, where each specialist strategy is capable of outperforming different opponents, the population as a whole can have a significantly higher generalization performance compared to individual strategies.
\end{abstract}

Index Terms-Coevolutionary learning, diversity maintenance, evolutionary computation (EC), generalization performance, iterated Prisoner's dilemma (IPD).

\section{INTRODUCTION}

$\mathbf{E}$ VOLUTIONARY COMPUTATION (EC) refers to the study of computational approaches that are motivated from and emulate the process of natural evolution. Although

Manuscript received April 20, 2009; revised July 14, 2009; accepted September 18, 2009. First published October 13, 2009; current version published December 01, 2009. The work of X. Yao was supported in part by EPSRC under Grant GR/T10671/01.

S. Y. Chong is with the School of Computer Science, University of Nottingham, Malaysia Campus (UNMC), 43500 Semenyih, Malaysia, and also with the Automated Scheduling, Optimisation and Planning (ASAP) Research Group, School of Computer Science, University of Nottingham, Nottingham NG8 1BB, U.K. (e-mail: Siang-Yew.Chong @ nottingham.edu.my).

$\mathrm{P}$. Tiňo and X. Yao are with the School of Computer Science, The University of Birmingham, Birmingham B15 2TT, U.K. (e-mail: P.Tino@cs.bham.ac.uk; X.Yao@cs.bham.ac.uk).

Digital Object Identifier 10.1109/TCIAIG.2009.2034269 current EC studies have since expanded and involved interdisciplinary research with other computational methodologies (such as neural networks) [1], [2], EC traditionally studies and still continues to focus on a broad class of population-based, stochastic search algorithms known as evolutionary algorithms (EAs) [3]-[5]. EAs can be described using the unified generate-and-test framework for search algorithms [2]. The search process of EAs involves samples (populations) of potential solutions drawn from the space of representation of the problem domain, which undergo iterated applications of variation (generation of new solutions based on solutions in the previous iteration through some perturbation processes) and selection (testing solutions for inclusion in the next iteration based on some quality or fitness measurements) [2].

Although EC has been studied in many problem domains (such as optimization [6], [7] and classification [8], [9]), games have played important roles in the development and understanding of EC [10]. A game has a specific set of rules that constrains strategies to certain behaviors (legal moves), with goals for strategies to meet (to win the game), and rewards for those that better achieve the goals under constraints of finite resources (payoff for a move). A game has enough subtleties to allow representation of a wide range of complex behaviors (a diverse set of strategies) [11]. Games can capture intrinsic properties of complex, real-world problems where EC methodologies are developed to obtain solutions, which can range from solutions (strategies) for board games to economic games [11], [12], but are sufficiently simple to enable extensive and in-depth analysis of EC methodologies.

In EC, coevolutionary learning refers to a broad class of population-based, stochastic search algorithms that involve the simultaneous evolution of competing solutions with coupled fitness [3]. As with EAs, coevolutionary learning can be described using the generate-and-test framework. For example, early coevolutionary learning systems are implemented using coevolutionary algorithms derived from EAs [11], [13]. However, coevolutionary learning and EAs are fundamentally different in how the fitness of a solution is assigned, which may lead to significantly different outcomes when they are applied to similar problems (different search behaviors on the space of solutions) [14], [15]. Classical EAs are formulated in the context of optimization [3], [5], [6], where an absolute fitness function is used to assign fitness values to solutions. The solution fitness is $o b$ jective (remains the same regardless of the composition of the population). Coevolutionary learning assigns a solution's fitness through its interactions with other competing solutions in the population. The solution fitness is subjective (depends on the composition of the population). 
Coevolutionary learning is originally proposed as a viable alternative for problems where it is very difficult to construct an absolute quality measurement for solutions (the fitness function) through which optimization-based search algorithms such as EAs can be used. The evolutionary search is guided by the strategic interactions between the competing solutions in the population. In particular, these interactions provide the basis to compute relative fitness values that are used to rank the competing solutions, which would affect the selection process of the population and subsequently the variation in the population. The potential for problem solving through coevolutionary learning can be realized from an arms-race dynamic in the evolutionary search process that leads to solutions that are innovative and of increasingly higher quality [16]-[20].

In coevolutionary learning, games have played an important role not only in the development of various coevolutionary algorithms, but also in understanding its approach to problem solving. In particular, games provide a natural framework to study coevolutionary learning as the interactions between the coevolving solutions in the population can be framed as game playing. Games are used to study two main properties of a coevolutionary learning system: decision making and learning [11]. Decision making refers to the ability of the evolved strategy to make appropriate responses for the given stimuli (behaviors) in light of specific goals that must be achieved. Learning refers to the ability of the system to train (evolve) strategies that can respond to a wide range of environments (opponents). The measure of success of a coevolutionary learning system in solving games can be viewed as its ability to evolve strategies with behaviors such that they can outperform against a large number of different opponents.

Although coevolutionary learning has been successfully applied in solving games [11], [13], [20]-[23], the methodology has been shown to suffer from coevolutionary pathologies that can potentially have a negative impact on its performance [19], [24]-[28]. In the context of game playing, poor search performance in coevolutionary learning can be a result of the overspecialization of the population to a particular strategy that performs well only against specific opponents rather than a large number of different opponents [26], [27].

As such, there are strong motivations to investigate and understand the conditions in the search process of coevolutionary learning that have impact on its performance. Generalization performance and diversity are two important and related issues of the study. For the first issue, one is concerned with the coevolutionary learning of strategies with high generalization performance, that is, strategies that can outperform against a large number of test strategies (opponents) that may not have been seen during coevolution [26], [29], [30]. For the second issue, one is concerned with diversity levels in coevolutionary learning [25], [27], [31].

The lack of diversity has been claimed to have a negative impact on the generalization performance of coevolutionary learning. Studies that have investigated this issue have proposed diversity maintenance techniques to introduce and maintain diversity in the population through specific use of selection and variation processes, which have been shown subsequently to improve the performance of coevolutionary learning [27],
[29], [31], [32]. However, it is not known whether there is a relationship between generalization performance and diversity in coevolutionary learning. Establishing any direct relationship between generalization performance and diversity theoretically would be very difficult. Although earlier studies have turned to various empirical approaches, there is a lack of rigorous analysis to investigate this issue in terms of generalization performance measures in coevolutionary learning [29], [32], [33]. Furthermore, these past studies made little attempt to measure diversity levels in coevolutionary learning despite strong claims that they are increased while investigating their impact on performance [24], [25], [29], [33].

In this paper, we present a detailed empirical study as a first step to investigate whether there is a relationship between generalization performance and diversity in coevolutionary learning. We have systematically investigated the impact of various diversity maintenance techniques on the generalization performance of coevolutionary learning quantitatively through case studies involving iterated prisoner's dilemma (IPD) games. Our case studies address the two shortcomings found in previous empirical studies. First, we have made a series of quantitative measurements of the generalization performance of coevolutionary learning systems with and without diversity maintenance that are based on the estimation procedure we have developed theoretically in [30]. Second, we have made a series of quantitative measurements of diversity levels in the population of various coevolutionary learning systems with and without diversity maintenance that are based on relevant diversity measures that have been identified earlier in [34]. These measurements allow us to determine whether the generalization performance and diversity levels in the population have been increased as a result of the application of diversity maintenance in coevolutionary learning in comparison to the classical coevolutionary learning (CCL) without diversity maintenance that we have used as a baseline.

In investigating the relationship between generalization performance and diversity in coevolutionary learning, we try to answer two related questions: 1) what is the right form of diversity that would be beneficial for generalization, and 2) what amount of the "right" form of diversity is needed for good generalization? The answers to these two questions may allow us to identify specific conditions whereby diversity can be exploited to improve the generalization performance in coevolutionary learning. Although our results have shown that not all diversity maintenance techniques that introduce and maintain diversity lead to a significant increase in the generalization performance of coevolutionary learning, we have identified a condition with which diversity plays a positive role in improving generalization performance. In particular, if individual strategies from the population can be combined (e.g., using a gating mechanism), there is the potential of exploiting diversity in coevolutionary learning to improve generalization performance. Specifically, when the introduction and maintenance of diversity lead to a speciated population during coevolution, where each specialist strategy is capable of outperforming different opponents, the population as a whole can have a significantly higher generalization performance compared to individual strategies.

The rest of this paper is organized as follows. Section II reviews a number of diversity maintenance techniques in coevo- 
lutionary learning that have been proposed in the past. The case studies that are presented in this paper compare coevolutionary learning employing these diversity maintenance techniques with CCL without diversity maintenance. Section III presents the methodology of the experiments used in the case studies. The section also describes how generalization performance and diversity levels of coevolutionary learning are measured. Section IV presents results of the case studies and discusses the observations made from the results. Section V concludes the paper with remarks for future studies.

\section{DiVERSity MAINTENANCE TECHNIQUES}

The search process in evolutionary systems can be described with the notion of how these systems maintain a spread of points (the population) in the search space through exploration and exploitation. An evolutionary system operating during an exploration phase would spend more effort searching other globally promising regions of the search space that are not covered by the current population. In contrast, evolutionary systems operating during an exploitation phase would spend more effort searching locally in regions covered by the current population to focus on obtaining the required solutions to the problem. The success of an evolutionary search depends on how and when to explore or exploit the search space so that solutions that are of better quality are obtained in subsequent generations. There may be a tradeoff between exploring and exploiting the search space in any generation, and understanding this tradeoff may lead to the design of adaptive control in the search process that leads to improvements in the search performance [35].

One common approach used to study the dynamics of the evolutionary process and the role they play in search is with the notion of diversity. Diversity is concerned with the levels and types of variety in the population [34], which can change depending on the exploration or exploitation phase of the evolutionary process. Many studies [36]-[43] have investigated methods that exploit diversity to further improve the search performance of evolutionary systems. Some of these algorithms are designed to introduce and maintain diversity in the population during the evolutionary search process. Early examples of these diversity maintenance techniques have been used in EAs to solve difficult optimization problems involving multiple optima [36]-[40]. The techniques have since been adapted for coevolutionary learning with the motivation that diversity may encourage fitness variations in the population for continued search of solutions with higher generalization performance [29], [44].

In our case studies, we categorize the different diversity maintenance techniques into two groups that are based on how the specific algorithm operates in the coevolutionary learning framework. Implicit techniques emphasize diversity maintenance through the selection process. Explicit techniques emphasize diversity maintenance through the variation process. This section describes several established diversity maintenance techniques in coevolutionary learning studies that we use for our case studies.

For clarity, we use examples of game playing in our description of how a specific diversity maintenance technique is implemented in coevolutionary learning. The interactions between solutions in coevolutionary learning that is used to determine the solution fitness (relative to other solutions) can be framed as game playing. A game is played between two strategies (solutions) and consists of a series of moves that are made by each strategy. During one game play, both strategies accumulate payoffs that are based on the joint moves they have made, which are then used to determine the outcome when the game finishes. An example would be the case where a strategy wins the game when it has a higher total payoff in comparison to the other strategy, which loses the game. Diversity maintenance techniques differ in terms of how information from game outcomes is obtained and used.

\section{A. Implicit Diversity Maintenance}

1) Speciation: One of the early implicit diversity maintenance techniques is speciation (or niching). Speciation is usually implemented with fitness sharing algorithms. The main idea of fitness sharing is to change fitness evaluations so that fitnesses of solutions are reduced to lower values if the solutions are more similar. Fitness sharing of solutions can be implemented by computing the shared fitness $f_{i}^{s}$ from the original, unshared fitness $f_{i}$ for each solution $i$ in the population [40]. This is to emphasize the search for more dissimilar solutions and possibly leading to a population forming a diverse set of niches with unique solutions. Competitive fitness sharing [27] and implicit fitness sharing [29] are two fitness sharing approaches that have been studied for coevolutionary learning.

In the competitive fitness sharing approach, each evolved strategy $i$ in the population would interact with a random set of opponent strategies $P_{x}$ sampled from the same population. Strategy $i$ plays against an opponent $j$ in $P_{x}$ and receives a fitness value of $1 / N_{j}$ if $i$ wins. $N_{j}$ is the number of opponents in the population that have defeated $j . N_{j}$ can be precomputed for each strategy $j$ in the population through a tournament where every strategy $j$ plays against all strategies in the population. Obviously, no strategy will win against a copy of itself, which means that the maximum value of $1 / N_{j}$ is $1 / 1=1$. The minimum value for the worst strategy that loses to all strategies in the population would be $1 / N_{j}=1 /$ POPSIZE (POPSIZE is the total number of parent and offspring strategies). The shared fitness for a strategy $f_{i}^{s}$ is calculated as follows:

$$
f_{i}^{s}=\sum_{j \in P_{x}} \frac{1}{N_{j}} \cdot O(i, j)
$$

where $O(i, j)$ is 1 if $i$ wins against $j$, and 0 otherwise. Essentially, the competitive fitness sharing approach encourages the selection of strategies that are unique in terms of defeating specific opponents that few other competing strategies could defeat (having higher fitness value of $1 / N_{j}$ as $N_{j}$ is smaller). These strategies are rewarded with a higher total sum of fitness values even though they may defeat a smaller number of opponents.

In the implicit fitness sharing approach, the shared fitness for each strategy $i$ in the population is obtained using the algorithmic procedure outlined in Fig. 1. During one typical run of the procedure for a strategy $i$ (there are $C$ number of runs), a random sample $\sigma$ of opponent strategies (other than strategy $i$ itself) would be drawn from the current population. The strategy 
Repeat $C$ times

1) Select a random sample $\sigma$ of opponent strategies from the current population.

2) Find the opponent strategy in $\sigma$ that achieves the highest payoff (or the largest winning margin) against $i$.

3) The best opponent strategy in $\sigma$ receives a fitness value (equivalent to the game payoff). In the case of a tie between opponent strategies, the fitness is shared equally.

Fig. 1. Procedure for implicit fitness sharing to assign shared fitness to a strategy.

$i$ would play against each opponent strategy in $\sigma$. The opponent that achieves the highest game payoff against strategy $i$ would receive a fitness value (we use the game payoff itself). If $n \leq|\sigma|$ number of opponents tie in terms of their game payoffs against strategy $i$, they would share the fitness (the game payoff divided by $n$ ). The approach has been shown mathematically to approximate the original fitness sharing scheme [45] but with the advantage of not having to use a sharing function, which can be problem specific [46].

2) Pareto Coevolution: Pareto coevolution is a coevolutionary learning approach with implicit diversity maintenance based on the framework of multiobjective optimization introduced earlier in EAs [24], [32], [33]. Pareto coevolution is motivated to address the problem associated with the use of scalar fitness evaluations in CCL. In the context of games, it has been argued that a strategy's fitness based on a simple weighted sum of values given by game outcomes can be misleading. One may not know at the time fitness evaluations are made a priori whether all outcomes are of equal importance (uniform weightings) or that some outcomes are more important (nonuniform weightings).

Pareto coevolution reformulates the fitness evaluation by explicitly using opponent strategies (test cases) to represent the different, underlying objectives of the problem that a strategy must address. The selection process in Pareto coevolution is based on the Pareto-dominance relationship. For single-population Pareto coevolution, to determine whether strategy $i$ Pareto dominates strategy $j$, we consider all $k \neq i, j$ strategies from the population as the $N$ objectives. The objective value $O(i, k)$ refers to the game outcome of strategy $i$ when it plays against strategy $k$. Strategy $i$ Pareto dominates $j$ if $i$ has at least the same game outcome as $j$ for every one of the $N$ objectives, and there is at least one objective for which $i$ has a better game outcome than $j$ [24]

$\operatorname{dom}(i, j) \Longleftrightarrow \forall k: O(i, k) \geq O(j, k) \wedge \exists k: O(i, k)>O(j, k)$

with $1 \leq k \leq N$. Strategies $i$ and $j$ are mutually nondominating if $i$ is better than $j$ on at least one objective and $j$ is better than $i$ on at least another objective, with both strategies being equivalent in the remaining objectives.

The selection process in Pareto coevolution can take the form of a ranking of strategies according to the Pareto layers that are obtained from the Pareto-dominance relationship. For example, mutually nondominating strategies in the Pareto front would be the most preferable strategies for selection. The second Pareto layer can be obtained by first removing strategies in the Pareto front from the population. The second Pareto layer would contain mutually nondominating strategies among the subpopulation that is obtained after removing the Pareto front from the full population. The following Pareto layers can be obtained systematically with respect to subpopulations that do not include strategies in higher Pareto layers [32].

3) Reducing Virulence: Both speciation and Pareto coevolution approaches to diversity maintenance require sophisticated algorithmic implementations. In [25], a simple approach that is inspired from the natural interactions between hosts and parasites was proposed. In a biological coevolutionary system, highly virulent parasites could potentially incapacitate the hosts, which in turn, reduce the chances for parasites to reproduce. In coevolutionary learning, similar observations have been made whereby strong competing solutions can quickly take over the population, leading to coevolutionary disengagement where the system is unable to differentiate between the solutions for continued evolutionary search [25].

The implementation of reducing virulence involves a form of rescaling of fitness values. CCL operates at maximum virulence. The original fitness value $f_{i}$ is assumed to be proportional to the solution $i$ 's ability to solve as many test cases as possible in the population (e.g., a simple weighted sum, which is normalized according to the fitness value of the best solution). In the context of game playing, one form of reducing virulence is to lower the fitness of the top performing strategies in the coevolving population that outperform the largest number of the opponents. Following [25], the virulence level $v$ is defined as the tendency to maximize fitness and a reduced virulence (RV) level $v$ changes an evolved strategy's original fitness value to

$$
f_{i}^{v}=\frac{2 f_{i}}{v}-\frac{f_{i}^{2}}{v^{2}}
$$

where $0.5 \leq v \leq 1.0$.

A coevolutionary learning system with maximum virulence level uses $v=1$, while a system with RV level has $v$ in the range of $[0.5,1.0]$ (e.g., moderate virulence level $v=0.75$ ). Fig. 2 compares how fitness values are rescaled between the use of maximum and moderate virulence levels. Note that the lowest value for $v$ is 0.5 as any lower value will indicate fitness is rescaled to encourage strategies to outperform smaller numbers of opponents [25].

\section{B. Explicit Diversity Maintenance}

Explicit diversity maintenance techniques involve increasing the level of variation in the population through variation operators in the coevolutionary search process. A simple approach 

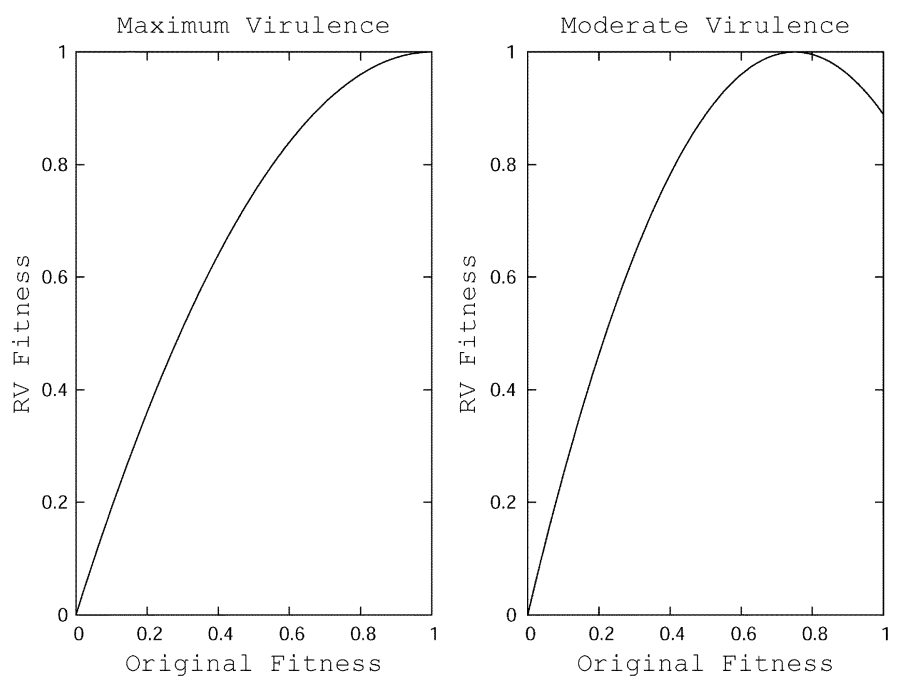

Fig. 2. Comparison of virulence curves between maximum virulence $(v=$ $1.0)$ and moderate virulence $(v=0.75)[25]$.

is to use a higher value for the parameter used in the variation operator that can increase the mutation probability. This simple approach is investigated in our case studies. However, it is noted that there are other more sophisticated approaches where the parameter controlling mutation probability can be varied according to some algorithmic procedures, e.g., setting higher parameter values to introduce and maintain more variations in the population if measurements on the population indicate low diversity levels [43].

\section{RELATIONSHIP BETWEEN GENERALIZATION PERFORMANCE AND DIVERSITY IN COEVOLUTIONARY LEARNING}

In this section, we present a methodology to investigate whether there is a relationship between generalization performance and diversity in coevolutionary learning. We use case studies to systematically investigate the impact of various diversity maintenance techniques on the generalization performance of coevolutionary learning quantitatively. The problem of the IPD game is considered. Two categories of diversity maintenance techniques that we investigate are: 1) implicit diversity maintenance techniques, which include speciation (competitive and implicit fitness sharing), Pareto coevolution, and reducing virulence; and 2) explicit diversity maintenance techniques with different and higher parameter settings for mutation probability.

\section{A. The IPD Game}

In the classical IPD game setting, two players engaged in repeated interactions are given two choices to make, cooperate and defect [47]-[49]. The classical IPD game is formulated using a predefined payoff matrix that specifies the payoff that a player receives for the joint moves of the player and the opponent. Fig. 3 illustrates the payoff matrix for the classical IPD game. Both players receive $R$ (reward) units of payoff if both cooperate. They both receive $P$ (punishment) units of payoff if they both defect. However, when one player cooperates while the other defects, the cooperator will receive $S$ (sucker) units

\begin{tabular}{|c|lr|lr|}
\hline & \multicolumn{2}{|c|}{ Cooperate } & \multicolumn{2}{|c|}{ Defect } \\
\hline Cooperate & & $\mathrm{R}$ & & $\mathrm{T}$ \\
& $\mathrm{R}$ & & $\mathrm{S}$ & \\
\hline Defect & & $\mathrm{S}$ & & $\mathrm{P}$ \\
& $\mathrm{T}$ & & $\mathrm{P}$ & \\
\hline
\end{tabular}

Fig. 3. Payoff matrix framework of a two-player, two-choice game. The payoff given in the lower left-hand side corner is assigned to the player (row) choosing the move, while that of the upper right-hand side corner is assigned to the opponent (column).

\begin{tabular}{cc|cccc} 
& & \multicolumn{4}{|c}{ PLAYER B } \\
& +1 & $+\frac{1}{3}$ & $-\frac{1}{3}$ & -1 \\
\hline \multirow{2}{*}{ PLAYER A } & +1 & 4 & $2 \frac{2}{3}$ & $1 \frac{1}{3}$ & 0 \\
& $-\frac{1}{3}$ & $4 \frac{1}{3}$ & 3 & $1 \frac{2}{3}$ & $\frac{1}{3}$ \\
& -1 & $5 \frac{2}{3}$ & $3 \frac{1}{3}$ & 2 & $\frac{2}{3}$ \\
& -1 & $3 \frac{2}{3}$ & $2 \frac{1}{3}$ & 1
\end{tabular}

Fig. 4. Payoff matrix for the two-player four-choice IPD game [50]. Each element of the matrix gives the payoff for player A.

of payoff while the defector receives $T$ (temptation) units of payoff.

In the classical IPD, the values $R, S, T$, and $P$ must satisfy the constraints: $T>R>P>S$ and $R>(S+T) / 2$. Any set of values can be used as long as they satisfy the IPD constraints (we use $T=5, R=4, P=1$, and $S=0$ in all our experiments). The game is played when both players choose between the two alternative choices over a series of moves. The dilemma in the game is reflected by the tension between rationality of individuals who are tempted to defect and rationality of the group where every individual is rewarded by mutual cooperation since both players who are better off mutually cooperating than mutually defecting are vulnerable to exploitation by the party who defects [49].

The classical IPD game has been extended to more complex versions to better model real-world interactions. One example is to extend the classical IPD with multiple, discrete levels of cooperation [26], [50]-[53]. The $n$-choice IPD game can be defined by the payoffs obtained through the following linear interpolation:

$$
p_{\mathrm{A}}=2.5-0.5 c_{\mathrm{A}}+2 c_{\mathrm{B}}, \quad-1 \leq c_{\mathrm{A}}, c_{\mathrm{B}} \leq 1
$$

where $p_{\mathrm{A}}$ is the payoff to player $\mathrm{A}$, given that $c_{\mathrm{A}}$ and $c_{\mathrm{B}}$ are the cooperation levels of the choices that players A and B make, respectively. Fig. 4 [50] illustrates the payoff matrix for the fourchoice IPD game we use in the case studies.

It is noted that when generating the payoff matrix for an $n$-choice IPD game, the following conditions must be satisfied [50].

1) For $c_{\mathrm{A}}<c_{\mathrm{A}}^{\prime}$ and constant $c_{\mathrm{B}}: p_{\mathrm{A}}\left(c_{\mathrm{A}}, c_{\mathrm{B}}\right)>p_{\mathrm{A}}\left(c_{\mathrm{A}}^{\prime}, c_{\mathrm{B}}\right)$.

2) For $c_{\mathrm{A}} \leq c_{\mathrm{A}}^{\prime}$ and $c_{\mathrm{B}}<c_{\mathrm{B}}^{\prime}$ : $p_{\mathrm{A}}\left(c_{\mathrm{A}}, c_{\mathrm{B}}\right)<p_{\mathrm{A}}\left(c_{\mathrm{A}}^{\prime}, c_{\mathrm{B}}^{\prime}\right)$.

3) For $c_{\mathrm{A}}<c_{\mathrm{A}}^{\prime}$ and $c_{\mathrm{B}}<c_{\mathrm{B}}^{\prime}: p_{\mathrm{A}}\left(c_{\mathrm{A}}^{\prime}, c_{\mathrm{B}}^{\prime}\right)>(1 / 2)$ $\left(p_{\mathrm{A}}\left(c_{\mathrm{A}}, c_{\mathrm{B}}^{\prime}\right)+p_{\mathrm{A}}\left(c_{\mathrm{A}}^{\prime}, c_{\mathrm{B}}\right)\right)$.

These conditions are analogous to those for the classical IPDs. The first condition ensures that defection always pays more. The second condition ensures that mutual cooperation 


\begin{tabular}{cc|cccc} 
& & \multicolumn{4}{|c}{ Opponent's Previous Move } \\
& & +1 & $+\frac{1}{3}$ & $-\frac{1}{3}$ & -1 \\
\hline \multirow{3}{*}{ Player's } & +1 & $m_{11}$ & $m_{12}$ & $m_{13}$ & $m_{14}$ \\
Previous Move & $-\frac{1}{3}$ & $m_{21}$ & $m_{22}$ & $m_{23}$ & $m_{24}$ \\
& -1 & $m_{31}$ & $m_{32}$ & $m_{33}$ & $m_{34}$ \\
& $m_{41}$ & $m_{42}$ & $m_{43}$ & $m_{44}$
\end{tabular}

Fig. 5. Direct lookup table representation for the deterministic and reactive memory-one IPD strategy that considers four choices (also includes $m_{\mathrm{fm}}$ for the first move, which is not shown in the figure).

results in higher payoff than that of mutual defection. The third condition ensures that alternating between cooperation and defection pay less in comparison to just playing cooperation.

Many past studies using IPD games were motivated to investigate specific conditions that can lead to the evolution of certain behaviors, for example, how and why cooperative behaviors can be learned through a coevolutionary process guided by interactions between competing strategies [54]-[59]. In this paper, our use of IPD games is motivated to determine whether diversity can have an impact on the coevolutionary learning of strategies that are "robust."

The notion of robustness has been defined in the context of strategy performance against some expert-designed strategies [13] and in the context of generalization of strategies obtained from a learning process [31], [44], [60]. Other studies such as in [61] have investigated in the context of nonlocal adaptation performance between populations of strategies. In this paper, we consider the notion of generalization and apply the theoretical framework of generalization performance in coevolutionary learning that we have formulated earlier in [30]. We consider the four-choice IPD game with 150 rounds for deterministic and reactive, memory-one strategies as it provides a difficult search problem (a large search space that cannot be exhaustively searched) for coevolutionary learning but one which we can analyze quantitatively.

\section{B. Strategy Representation}

For the IPD games involving deterministic and reactive strategies, several representations have been investigated in the past, which include a lookup table with bit-string encoding [13], finite-state machines [55]-[57], and neural networks [50], [53], [62], [63]. In this paper, we use the direct lookup table strategy representation that we have introduced and studied in [50], which directly represents IPD strategy behaviors through a one-to-one mapping between the genotype space (strategy representation) and the phenotype space (behaviors).

Fig. 5 illustrates the direct lookup table representation for the IPD strategies with four choices and memory-one that we consider in the case studies. The elements of the direct lookup table $m_{i j}, i, j=1, \ldots, n$, specify choices that are to be made given the inputs $i$ (player's own previous choice) and $j$ (opponent's previous choice). Instead of using pregame inputs (two for memory-one strategies), the first move is specified independently $m_{\mathrm{fm}}$. For the four-choice IPD (Fig. 5), each element can take values of $+1,+1 / 3,-1 / 3$, and -1 . These values are the same as those that are used to generate the payoffs in the payoff matrix (Fig. 4) through a linear interpolation (4).
A simple mutation-based variation operator is used to generate an offspring from a parent strategy when using the direct lookup table for strategy representation [50]. For the $n$-choice IPD, mutation replaces the original choice of a particular element in the direct lookup table with one of the other remaining $n-1$ possible choices with an equal probability of $1 /(n-1)$. Each table element has a fixed probability $p_{m}$ of being replaced by one of the remaining $n-1$ choices. The mutation-based variation operator can provide sufficient variations on strategy behaviors directly with the use of the direct lookup table representation (even for the more complex IPD game with more choices) [50].

The important advantage to using the direct lookup table representation is that the search space (given by the strategy representation) is the same as the strategy space (assuming uniform strategy distribution in the strategy space) [30]. This simplifies and allows direct investigation to be made on the conditions in a coevolutionary learning search process that can have impact on the generalization performance. Here, variation and selection operators can directly affect the learning of strategy behaviors from the use of the direct lookup table representation. This in turn allows us to investigate the impact of both explicit and implicit diversity maintenance techniques on the coevolutionary learning of strategy behaviors. Furthermore, we can measure (estimate) the generalization performance of evolved strategies using a random sample of test strategies [30].

\section{Coevolutionary Learning}

All coevolutionary learning systems that we investigate share the same generate-and-test framework guided by strategic interactions through IPD game play. The basic coevolutionary learning system without any specific application of diversity maintenance (our baseline) is CCL, which is implemented through the following procedure [50].

1) Generation step $t=1$ : Initialize POPSIZE/2 parent strategies $P_{i}, i=1,2, \ldots$, POPSIZE $/ 2$, randomly.

2) Generate POPSIZE $/ 2$ offspring $O_{i}, i=1,2, \ldots$, POPSIZE $/ 2$, from POPSIZE/2 parents using a variation operator.

3) All pairs of strategies compete, including the pair where a strategy plays itself (round-robin tournament). For POPSIZE strategies in a population, every strategy competes a total of POPSIZE games.

4) Select the best POPSIZE/2 strategies based on total payoffs of all games played. Increment generation step $t \leftarrow t+1$.

5) Steps 2)-4) are repeated until a termination criterion (a fixed number of generation) is met.

The settings for classical coevolutionary learning system (CCL-PM05) that we use as baseline for comparison with coevolutionary learning systems with specific application of diversity maintenance are summarized in Table I.

In implementing coevolutionary learning systems with diversity maintenance, changes are made only to the affected variation or selection operators. This is to facilitate a more direct comparison of the impact of different diversity maintenance techniques on coevolutionary learning, whether explicitly through the variation process, or implicitly through the selection process. Note that different implicit diversity maintenance techniques would rank strategies in the population differently, 
TABLE I

SUMMARY OF SETTINGS USED IN CCL (BASELINE)

\begin{tabular}{|l||l|}
\hline $\begin{array}{l}\text { Strategy Representation } \\
\text { Variation Operator }\end{array}$ & $\begin{array}{l}\text { Direct Look-up Table } \\
\text { Simple mutation of direct look-up table } \\
\text { with probability of mutation, } p_{m}=0.05\end{array}$ \\
Population Structure & $\begin{array}{l}\text { Single-population } \\
\text { POPSIZE }\end{array}$ \\
$\begin{array}{l}\text { Fitness Evaluation } \\
\text { Selection }\end{array}$ & $\begin{array}{l}\text { Round-robin tournament } \\
\text { Best-half of the population after ranking, }(\mu, \lambda) \text { EP } \\
\text { Selection Scheme }\end{array}$ \\
Generation & 600 \\
\hline
\end{tabular}

TABLE II

Summary of IMPlicit Diversity MAINTENANCE TeChNiQueS USED IN THE EXPERIMENTS

\begin{tabular}{|l||l|}
\hline \multicolumn{1}{|c||}{ CFSD } & $\begin{array}{l}\text { Competitive fitness sharing with disassortative sampling } \\
\text { Fitness values obtained using Equation 1 } \\
\text { Opponent sample size of } 5\end{array}$ \\
\hline CFSR & $\begin{array}{l}\text { Competitive fitness sharing with random sampling } \\
\text { Fitness values obtained using Equation 1 } \\
\text { Opponent sample size of } 5\end{array}$ \\
\hline IFSD & $\begin{array}{l}\text { Implicit fitness sharing with disassortative sampling } \\
\text { Fitness values obtained using procedure outlined in Figure 1 } \\
\text { Opponent sample size of } 3 \text { and procedure repeated } 3 \text { times }\end{array}$ \\
\hline IFSR & $\begin{array}{l}\text { Implicit fitness sharing with random sampling } \\
\text { Fitness values obtained using procedure outlined in Figure 1 } \\
\text { Opponent sample size of } 3 \text { and procedure repeated } 3 \text { times }\end{array}$ \\
\hline PAR & $\begin{array}{l}\text { Pareto co-evolution } \\
\text { Ranking based on pareto dominance relation given by Equation } 2\end{array}$ \\
\hline RV & $\begin{array}{l}\text { Reduced virulence } \\
\text { Fitness values obtained using Equation } 3 \text { with } v=0.75\end{array}$ \\
\hline
\end{tabular}

and that the best half of the ranked population are selected as parents for the following generation. The implementation details have been described earlier in Section II, although we summarize further details such as the choices of parameters and differentiate between the various implicit diversity maintenance techniques in Table II.

For case studies involving coevolutionary learning with speciation, we investigate both competitive and implicit fitness sharing techniques. Furthermore, we investigate two sampling procedures to obtain the set of opponents that is used for fitness evaluation. The first procedure is disassortative sampling that first selects a random opponent from the current population before choosing successive opponents that are the most different (in terms of edit distance for the direct lookup table representation that we describe in more detail later) from the previous opponents [29]. The second procedure is simple random sampling [29].

For Pareto coevolution, the single-population approach that was proposed in [33] is used. This is to facilitate a more direct comparison with other coevolutionary learning systems we investigate in this study that use only one population. For the coevolutionary learning with RV, we consider the moderate virulence level that has been studied in detail earlier in [25].
For case studies involving the application of explicit diversity maintenance to coevolutionary learning, we consider CCL approach and investigate systems with higher parameter value settings for the probability of mutation $p_{m}$. We consider the CCL with $p_{m}=0.05$ (CCL-PM05) as the basic coevolutionary learning system (baseline) for comparison with other coevolutionary learning systems having diversity maintenance. We use other $p_{m}$ settings, i.e., $p_{m}=0.1$ (CCL-PM10), $p_{m}=0.15$ (CCL-PM15), $p_{m}=0.2$ (CCL-PM20), and $p_{m}=0.25$ (CCL-PM25), to investigate the impact of explicit diversity maintenance on the generalization performance of coevolutionary learning. In the case studies, all experiments involving different coevolutionary learning systems are repeated in 30 independent runs to allow for statistical analysis.

\section{Measuring Generalization Performance}

We have recently formulated a theoretical framework for measuring generalization performance in coevolutionary learning, and have applied the framework in the context of the coevolutionary learning of IPD games. The generalization performance of a strategy is defined as its average performance against test (opponent) strategies [30].

In formulating the generalization performance measure for coevolutionary learning, consider a game with the strategy space given by a set $\mathcal{S}$ of $M$ distinct strategies $\mathcal{S}=\{1,2, \ldots, M\}$. Let the selection of individual test strategies from $\mathcal{S}$ be represented by a random variable $J$ taking on values $j \in \mathcal{S}$ with probability $P_{\mathcal{S}}(j)$. Denote the game outcome of strategy $i$ playing against the test strategy $j$ by $G_{i}(j)$ (conversely, $G_{j}(i)$ is the game outcome of strategy $j$ against strategy $i$ ).

Different definitions of $G_{i}(j)$ (i.e., different notions of game outcomes) for the generalization performance indicate different measures of quality. The win-lose and average-payoff functions for $G_{i}(j)$ are two examples. Let $g(i, j)$ and $g(j, i)$ be the payoffs for strategies $i$ and $j$ at the end of the IPD game, respectively. Strategy $i$ wins the game if $g(i, j)>g(j, i)$, else $i$ loses the game. The win-lose function for $G_{i}(j)$ for the IPD game is defined as

$$
G_{\mathrm{W}}(i, j)= \begin{cases}C_{\mathrm{WIN}}, & \text { for } g(i, j)>g(j, i) \\ C_{\mathrm{LOSE}}, & \text { for otherwise }\end{cases}
$$

where $C_{\mathrm{WIN}}$ and $C_{\mathrm{LOSE}}$ are arbitrary constants with $C_{\mathrm{WIN}}>$ $C_{\mathrm{LOSE}}\left(\right.$ we use $C_{\mathrm{WIN}}=100$ and $C_{\mathrm{LOSE}}=0$ ).

In this paper, we consider the win-lose function as it simplifies the analysis of the experiments. In particular, we know the "all defect" strategy that plays full defection only to be the strategy with the maximum generalization performance when the win-lose function is used irrespective of how test strategies are distributed in $\mathcal{S}$ (this is not necessarily true for other definitions such as the average-payoff function). We arbitrarily choose a stricter form of the win-lose function to measure performance (since a loss is awarded to both sides in the case of a tie).

The true generalization performance of a strategy $i$ is defined as the mean performance against all test strategies

$$
G_{i}=\sum_{j=1}^{M} P_{\mathcal{S}}(j) G_{i}(j) .
$$


That is, $G_{i}$ is the mean of the random variable $G_{i}(J)$

$$
G_{i}=E_{P_{\mathcal{S}}}\left[G_{i}(J)\right]
$$

Some test strategies may be favored over the others, or all test strategies can be considered with equal probability a priori. In particular, when all strategies are equally likely to be selected, i.e., when $P_{\mathcal{S}}$ is uniform, we have

$$
G_{i}=\frac{1}{M} \sum_{j=1}^{M} G_{i}(j) .
$$

The size $M$ of the strategy space $\mathcal{S}$ can be very large, making direct estimation (6) of the generalization performance $G_{i}$ computationally infeasible. For example, in the case of the four-choice IPD game involving deterministic and reactive, memory-one strategies, $M=4^{4^{2}+1}=4^{17} \approx 1.7 \times 10^{10}$.

In practice, one can estimate $G_{i}$ using a random sample $S_{N}$ of $N$ test strategies drawn independent and identically distributed (i.i.d.) from $\mathcal{S}$ with probability $P_{\mathcal{S}}$. The estimated generalization performance of strategy $i$ is then

$$
\hat{G}_{i}\left(S_{N}\right)=\frac{1}{N} \sum_{j \in S_{N}} G_{i}(j)
$$

If the game outcomes $G_{i}(J)$ vary within a finite interval $\left[G_{\mathrm{MIN}}, G_{\mathrm{MAX}}\right]$ of size $R=G_{\mathrm{MAX}}-G_{\mathrm{MIN}}$, the variance of $G_{i}(J)$ is upper bounded by $\sigma_{\mathrm{MAX}}^{2}=\left(G_{\mathrm{MAX}}-G_{\mathrm{MIN}}\right)^{2} / 4=$ $R^{2} / 4$. Using Chebyshev's theorem [64], we obtain

$$
P\left(\left|\hat{G}_{i}-G_{i}\right| \geq \epsilon\right) \leq \frac{R^{2}}{4 N \cdot \epsilon^{2}}
$$

for any positive number $\epsilon>0$. Note that Chebyshev's bound is distribution free, e.g., no particular form of distribution of $G_{i}(J)$ is assumed. Equation (8) allows one to make a statistical claim about how confident one is about the accuracy of an estimate given a random test sample of a known size $N$.

Let $\left|D_{N}\right|=\left|\hat{G}_{i}-G_{i}\right|$. The inequality (8) can be rewritten as follows:

$$
P\left(\left|D_{N}\right|^{\prime} \geq \epsilon^{\prime}\right) \leq \frac{1}{4 N \cdot \epsilon^{\prime 2}}
$$

where $\left|D_{N}\right|^{\prime}=\left|D_{N}\right| / R$ and $\epsilon^{\prime}=\epsilon / R$. For the case studies, we use a sample of random test strategies to estimate the generalization performance of strategy $i$ obtained through coevolutionary learning $\hat{G}_{W}(i)$, based on IPD game outcomes defined by $G_{\mathrm{W}}(i, j)$. Given the high computation cost that is involved in estimating the generalization performance through a large number of IPD game plays, we consider a sample size of $N=100000$. Assuming $\epsilon^{\prime}=0.01$ (error at $1 \%$ of $R=$ 100), Chebyshev's bound gives $P\left(\left|D_{N}\right|^{\prime} \geq \epsilon^{\prime}\right) \leq 1 /(4 \times$ $\left.100000 \times 0.01^{2}\right)=0.025$. We can claim with a probability at least $1-0.025=0.975>0.95$ that the error for the generalization performance estimate satisfies $\left|D_{N}\right|^{\prime} \leq 0.01$.

In game playing, one may be more interested in estimating the generalization performance with respect to a biased test sample, e.g., biased towards test strategies that are more likely to be encountered in a real-world scenario such as game tournaments, rather than an unbiased sample where every test strategy is equally likely to be encountered. A biased test sample may consist of "good" strategies based on some performance criteria, e.g., outperforming a large number of test strategies. This biased sample of "good" test strategies can be obtained using a procedure we have introduced and studied earlier in [30].

1) Partial enumerative search run $r=1$ : sample PS strategies, $Q_{i}, i=1,2, \ldots, \mathrm{PS}$, randomly.

2) Every strategy competes with all other strategies in the sample, including its twin (i.e., each strategy competes in a total of PS games).

3) Detect the strategy index $s \in\{1,2, \ldots, \mathrm{PS}\}$ so that $Q_{s}$ yields the highest total payoff. Let $Q^{(r)}:=Q_{s}$. Increment run $r=r+1$.

4) Repeat steps 1)-3) PE times to obtain a PE-sized biased sample of test strategies, $Q^{(r)}, r=1,2, \ldots, \mathrm{PE}$.

The population (sample) size for each partial enumerative search is PS $=10^{6}$, which is significantly larger than the maximum number of unique strategies in an evolutionary run (PS $>$ (generation $\times$ POPSIZE)). This is so that the test strategy is: 1 ) likely not to have been encountered before by evolved strategies and 2) more challenging (having outperformed a significantly larger number of opponents compared to evolved strategies). The procedure is independently repeated $\mathrm{PE}=20$ times so that a more diverse sample of test strategies is obtained. A sample of PE test strategies obtained from a single partial enumerative search may be less diverse (they are behaviorally similar with the same weakness that can be exploited) and lower performing due to a poor population sample.

For the case studies, we estimate the generalization performance with respect to unbiased and biased samples of test strategies, which we denote as $\hat{G}_{W}$ and $\hat{G}_{W}^{B}$, respectively. Both estimates $\hat{G}_{W}(i)$ and $\hat{G}_{W}^{B}(i)$ can be computed for the current top performing evolved strategy $i$ in a particular generation. However, analysis on the generalization performance of coevolutionary learning system can be made by taking generalization performance estimates on the best $U$ evolved strategies from the population $\mathrm{SPOP}_{U}=\left(\operatorname{spop}_{1}, \ldots, \operatorname{spop}_{U}\right)$, which is an ordered set (ordered according to their internal fitness values).

- Best

- Average

$$
\operatorname{Best}\left(G_{\mathrm{SPOP}_{U}}\right)=\hat{G}_{\mathrm{spop}_{1}} .
$$

$$
\operatorname{Avg}\left(G_{\mathrm{SPOP}_{U}}\right)=\frac{1}{U} \sum_{l=1}^{U} \hat{G}_{\mathrm{spop}_{l}}
$$

- Population

$$
\operatorname{Pop}\left(G_{\mathrm{SPOP}_{U}}\right)=\frac{1}{N} \sum_{j \in S_{N}} \min \left(\sum_{l=1}^{U} G_{\text {spop }_{l}}(j), G_{\mathrm{MAX}}\right) \text {. }
$$

The "Population" measurement allows us to estimate the generalization performance of the coevolving population as a whole. For simplicity, we assume that the population can be combined with an ideal gating mechanism [29], [60]: it chooses the evolved strategy in $\mathrm{SPOP}_{U}$ that best performs against each of the test strategies. For example, given five test strategies, if spop $_{1}$ outperforms the first two test strategies, and $\mathrm{spop}_{2}$ outperforms the last three test strategies, the population of $\left\{\mathrm{spop}_{1}, \mathrm{spop}_{2}\right\}$ with this gating mechanism outperforms all test strategies (it will choose spop $_{1}$ for the first two test 
strategies, and $\mathrm{spop}_{2}$ for the others). As such, the "Population" measurement would obtain an upper bound on the generalization performance of the population. We use this measurement to compare the generalization performance of the population and an individual strategy by investigating their difference in the coverage of the whole strategy space (i.e., performance against all test strategies). This analysis allows us to investigate if diversity maintenance can have an impact on the coevolving population and subsequently be exploited for improvements in generalization performance.

For the case studies, $U=5$ top performing individual strategies are selected from the coevolving population. Estimates $\hat{G}_{W}$ and $\hat{G}_{W}^{B}$ are made for the top performing individual strategies in the population $\left(\operatorname{Best}\left(G_{\mathrm{SPOP}_{U}}\right)\right.$ and $\left.\operatorname{Avg}\left(G_{\mathrm{SPOP}_{U}}\right)\right)$ and for the population $\left(\operatorname{Pop}\left(G_{\operatorname{SPOP}_{U}}\right)\right)$.

\section{E. Measuring Diversity}

Different diversity measurements obtain different information on the levels and type of variety from the population of an evolutionary system. Some diversity measures may be more important in comparison to other diversity measures for specific problems. Here, the main motivation is to obtain some general information on the diversity levels for comparison between coevolutionary learning systems with and without diversity maintenance. For the case studies, we consider two different diversity measurements, i.e., genotypic and phenotypic diversity measurements. In an earlier study [34] that had conducted a systematic empirical investigation on various diversity measures, results suggested strong correlations between the diversity levels based on the genotypic and phenotypic diversity measurements with the fitness of the top performing solutions obtained from evolutionary search processes. The study concluded that these two diversity measurements may be useful in obtaining information on diversity levels that reflects the evolutionary search process with respect to its performance.

Genotypic diversity relates to the level of variety in the genotypic space (the search space that is specified by the solution representation). The genotypic diversity measurement that we use is based on the edit distance diversity measurement [34], [65], [66], which we have adapted to measure the edit distance of deterministic and reactive, memory-one IPD strategies with a direct lookup table representation. ${ }^{1}$ The edit distance between two strategies $i$ and $j$ is related to the minimum number of transformations that is required to change one strategy's direct lookup table to the other strategy's direct lookup table

$$
\operatorname{dist}_{\text {ed }}(i, j)=\frac{1}{n_{e}} \sum_{k=1}^{n_{e}} d\left(i_{k}, j_{k}\right)
$$

where $i_{k}$ and $j_{k}$ are the direct lookup table elements of strategies $i$ and $j$, respectively, and $d\left(i_{k}, j_{k}\right)$ is a distance metric on $i_{k}$ and $j_{k} . n_{e}$ is the total number of elements of the direct lookup table. For the four-choice IPD strategy, $n_{e}=17$ (one element for the first move and 16 elements for decisions based on joint past moves as shown in Fig. 5).

\footnotetext{
${ }^{1}$ Note that since the strategy representation has a fixed length data structure, we are measuring the Hamming distance. However, we use the term edit distance in this paper based on the references of past studies [34], [65], [66], and also to differentiate with the more common usage of the term Hamming distance in EAs using the binary string representation.
}

For the distance metric $d\left(i_{k}, j_{k}\right)$, we use the Levenshtein distance as in [67]

$$
d\left(i_{k}, j_{k}\right)= \begin{cases}0, & i_{k}=j_{k} \\ 1, & i_{k} \neq j_{k}\end{cases}
$$

Note that a transformation for a particular element is made only as a substitution from one choice to another. For example, if two strategies $i$ and $j$ differ only in the choice they make for the first move, e.g., +1 for strategy $i$ and $-1 / 3$ for strategy $j$, only one transformation is required to change one strategy's direct lookup table to the other strategy's direct lookup table (substituting +1 with $-1 / 3$ to transform the direct lookup table representation of strategy $i$ to that of strategy $j$ ). The edit distance between these two strategies is $1 / 17$.

The genotypic diversity measurement based on edit distance on a coevolving population can be obtained by taking the average value of pairwise distances of strategies in the population [68]

$$
\frac{2}{\text { POPSIZE(POPSIZE }-1)} \sum_{1 \leq i<j \leq \text { POPSIZE }} \operatorname{dist}_{\mathrm{ed}}(i, j) \text {. }
$$

Phenotypic diversity relates to the level of variety in the phenotypic space (e.g., fitness values used in the selection process of coevolutionary learning) [34], [69]. Studies such as in [70] have investigated fingerprints of strategies based on their behavioral responses against opponents. Here, we use the simple phenotypic diversity measurement based on the entropy diversity measurement [34], [35]

$$
-\sum_{k} p_{k} \cdot \log p_{k}
$$

where $p_{k}$ refers to the proportion of a particular fitness value in the population. In our case studies, the fitness value is the average IPD game payoff obtained from a round robin tournament with the entire population of coevolving strategies.

\section{RESUlts AND Discussion}

In this section, we present an empirical study to investigate whether there is a relationship between generalization performance and diversity in coevolutionary learning. Using case studies, we systematically investigate the impact of different diversity maintenance techniques to the coevolutionary learning of the four-choice IPD on: 1) generalization performance and 2) diversity. We use the results of CCL without diversity maintenance as a baseline for comparison with results of coevolutionary learning systems with diversity maintenance.

We first present the results of the case studies that investigate the impact of implicit diversity maintenance on the generalization performance of coevolutionary learning. This is followed by the results of case studies that investigate the impact of explicit diversity maintenance on the generalization performance of coevolutionary learning. After that, we present results on the case studies that investigate the impact of implicit and explicit diversity maintenance on the diversity levels of the coevolving population.

In general, we have made observations that are consistent with those from past studies on the impact of individual diversity 
maintenance techniques on the generalization performance or diversity (as some of the past studies have made actual measurements for only one quantity) for coevolutionary learning of the IPD game. More importantly, through the use of the case studies with common quantitative measurements for generalization performance and diversity, our results indicate that the introduction and maintenance of diversity do not necessarily lead to a significant increase in the generalization performance of coevolutionary learning. However, our observations and subsequent analysis of results have suggested the potential that diversity can be exploited in improving the generalization performance of coevolutionary learning if the coevolved strategies in the population can be combined (e.g., using a gating mechanism).

\section{A. Generalization Performance of Coevolutionary Learning With Implicit Diversity Maintenance}

We first present the results of the generalization performance measurements with respect to an unbiased sample $\hat{G}_{W}$. We compare the generalization performance of different coevolutionary learning systems with implicit diversity maintenance with $\mathrm{CCL}$ without diversity maintenance (CCL-PM05) for the four-choice IPD. Three measurements for $\hat{G}_{W}, \operatorname{Best}\left(G_{\mathrm{SPOP}_{U}}\right), \operatorname{Avg}\left(G_{\mathrm{SPOP}_{U}}\right)$, and $\operatorname{Pop}\left(G_{\mathrm{SPOP}_{U}}\right)$, are obtained. Table III summarizes the results of the measurements at the end of the coevolutionary learning process.

In general, mixed results are obtained for the comparison of $\hat{G}_{W}$ measurements of coevolutionary learning systems with different implicit diversity maintenance techniques and the coevolutionary learning without diversity maintenance (CCL-PM05). For example, the generalization performance of coevolutionary learning with RV is actually lower than that of CCL-PM05. Table III shows that all three measurements $\operatorname{Best}\left(G_{\mathrm{SPOP}_{U}}\right)$, $\operatorname{Avg}\left(G_{\mathrm{SPOP}_{U}}\right)$, and $\operatorname{Pop}\left(G_{\mathrm{SPOP}_{U}}\right)$ for $\hat{G}_{W}$ are lower for the case of RV in comparison to the case of CCL-PM05.

However, coevolutionary learning with competitive fitness sharing has resulted in significantly higher generalization performance compared to CCL-PM05. For example, Table III shows that both coevolutionary learning systems using competitive fitness sharing with disassortative sampling (CFSD) and competitive fitness sharing with random sampling (CFSR) have higher generalization performance in all three measurements $\operatorname{Best}\left(G_{\mathrm{SPOP}_{U}}\right), \operatorname{Avg}\left(G_{\mathrm{SPOP}_{U}}\right)$, and $\operatorname{Pop}\left(G_{\mathrm{SPOP}_{U}}\right)$ for $\hat{G}_{W}$ compared to the case of CCL-PM05 that is statistically significant. In comparing between the two sampling procedures the impact on the generalization performance of coevolutionary learning, there is little difference in the results for using disassortative sampling in CFSD and for using simple random sampling in CFSR.

For coevolutionary learning systems using implicit fitness sharing with disassortative sampling (IFSD) and implicit fitness sharing with random sampling (IFSR) and Pareto coevolution (PAR), Table III shows that only the comparisons for $\operatorname{Pop}\left(G_{\mathrm{SPOP}_{U}}\right)$ measurements for $\hat{G}_{W}$ with that of CCL-PM05 result in statistical significant differences. For both $\operatorname{Best}\left(G_{\mathrm{SPOP}_{U}}\right)$ and $\operatorname{Avg}\left(G_{\mathrm{SPOP}_{U}}\right)$ measurements for $\hat{G}_{W}$, the comparisons between coevolutionary learning with and without the particular implicit diversity maintenance do not reveal any statistical significant difference. The $\operatorname{Best}\left(G_{\mathrm{SPOP}_{U}}\right)$
TABLE III

Summary of the Results For the Generalization PERFormance With ReSPECT TO AN UnBIASEd SAMPLE GIVEn by Best $\left(G_{\text {SPOP }_{U}}\right)$, $\operatorname{Avg}\left(G_{\text {SPOP }_{U}}\right)$, AND $\operatorname{Pop}\left(G_{\text {SPOP }_{U}}\right)$ FOR DIFFERENT COEVOLUTIONARY LEARNING SYSTEMS WITH IMPLICIT DIVERSITY MAINTENANCE FOR THE FOUR-CHOICE IPD TAKEN AT THE FinAL GENERATION. For EACH SYSTEM, THE AVERAGE VALUE AND THE ERROR AT 95\% CONFIDENCE INTERVAL OVER 30 RUNS ARE Noted. VALUES IN BOLD INDICATE THAT THE PARTICULAR SySTEM HaS A SignifiCANTLY HighER DiVERSITY LEVEl COMPARED TO THAT OF CCL-PM05 BASED ON THE PAIRED WILCOXON SIGNED RANK TEST FOR Statistical Significance DifferenCE AT the 0.05 LeVEl OF SignificanCE

\begin{tabular}{|c||c|c|c|}
\hline Experiment & $\operatorname{Best}\left(G_{\mathrm{SPOP}_{U}}\right)$ & $\operatorname{Avg}\left(G_{\mathrm{SPOP}_{U}}\right)$ & $\operatorname{Pop}\left(G_{\mathrm{SPOP}_{U}}\right)$ \\
\hline CCL-PM05 & $61.56 \pm 5.91$ & $61.17 \pm 5.00$ & $73.67 \pm 4.56$ \\
CFSD & $\mathbf{8 9 . 9 8} \pm \mathbf{2 . 2 8}$ & $\mathbf{9 0 . 2 3} \pm \mathbf{1 . 2 7}$ & $\mathbf{9 5 . 0 2} \pm \mathbf{0 . 6 7}$ \\
CFSR & $\mathbf{8 8 . 6 4} \pm \mathbf{2 . 6 2}$ & $\mathbf{8 6 . 4 9} \pm \mathbf{2 . 1 4}$ & $\mathbf{9 5 . 0 1} \pm \mathbf{0 . 8 5}$ \\
IFSD & $55.26 \pm 7.42$ & $53.63 \pm 3.39$ & $\mathbf{9 1 . 6 8} \pm \mathbf{1 . 6 1}$ \\
IFSR & $49.63 \pm 6.89$ & $50.27 \pm 5.27$ & $\mathbf{7 9 . 0 1} \pm \mathbf{4 . 0 1}$ \\
PAR & $69.43 \pm 6.70$ & $66.96 \pm 5.11$ & $\mathbf{9 1 . 6 2} \pm \mathbf{3 . 3 7}$ \\
RV & $44.89 \pm 7.43$ & $47.72 \pm 7.31$ & $68.22 \pm 7.20$ \\
\hline
\end{tabular}

and $\operatorname{Avg}\left(G_{\mathrm{SPOP}_{U}}\right)$ measurements for both IFSD and IFSR are actually lower compared to that of CCL-PM05. For PAR, $\operatorname{Best}\left(G_{\mathrm{SPOP}_{U}}\right)$ and $\operatorname{Avg}\left(G_{\mathrm{SPOP}_{U}}\right)$ measurements are slightly higher in comparison to that of CCL-PM05.

The results for coevolutionary learning with implicit fitness sharing (IFSD and IFSR) are in agreement with previous claims made in [29] and [60]. The generalization performance of each individual evolved strategy is not necessarily improved (as indicated by lower $\operatorname{Best}\left(G_{\mathrm{SPOP}_{U}}\right)$ and $\operatorname{Avg}\left(G_{\mathrm{SPOP}_{U}}\right)$ measurement values in Table III). However, if each evolved strategy specializes against smaller but different groups of opponents, a population of these specialist strategies has a significantly higher generalization performance if the population can sufficiently often and consistently choose the best strategy from the available pool against the opponents through a gating mechanism (as indicated by significantly higher $\operatorname{Pop}\left(G_{\mathrm{SPOP}_{U}}\right)$ measurement values in Table III). In addition, the effect of speciation on the coevolving population is maintained throughout the evolutionary process for both IFSD and IFSR. Fig. 6 shows that the difference between the generalization performance measurements for individual evolved strategies and the population is maintained on average across the generations.

By taking plots of the average results over 30 independent runs for the generalization performance measurements, we can observe the overall generalization performance and how it changes as a result of applying a particular diversity maintenance technique in coevolution. For implicit fitness sharing, we observe in Fig. 6 a significant difference between the generalization performance of the population as a whole and individual strategies throughout coevolution. However, we also note some small degree of local fluctuations in the generalization performance particularly for the measurement of individual strategies. This is not surprising since the implicit fitness sharing technique aims to increase diversity directly (to promote speciation in the population), which would indirectly result in an increase in the generalization performance when the population of strategies can be combined using a gating mechanism. Although diversity is maintained in the coevolving population, it does not necessarily result in a well-speciated 


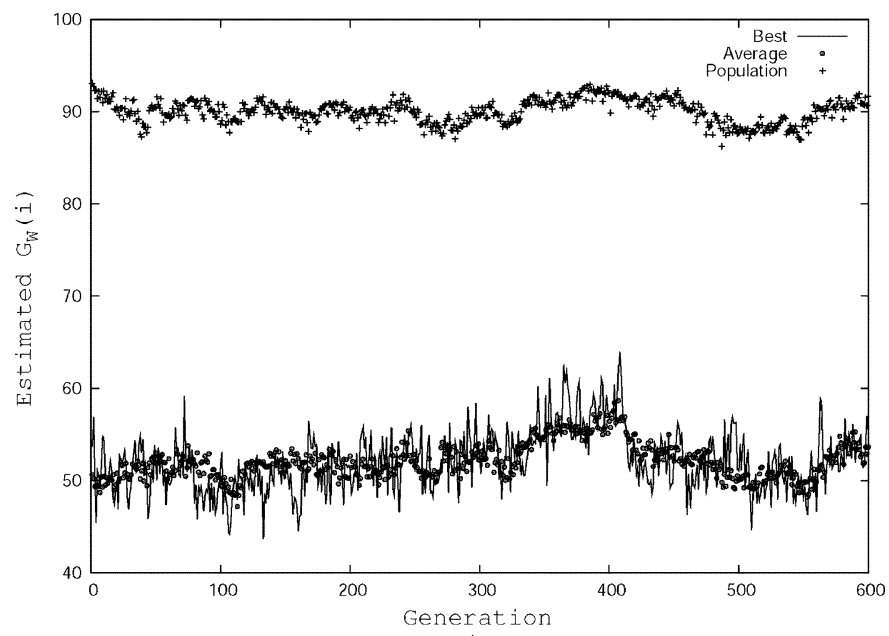

(a)

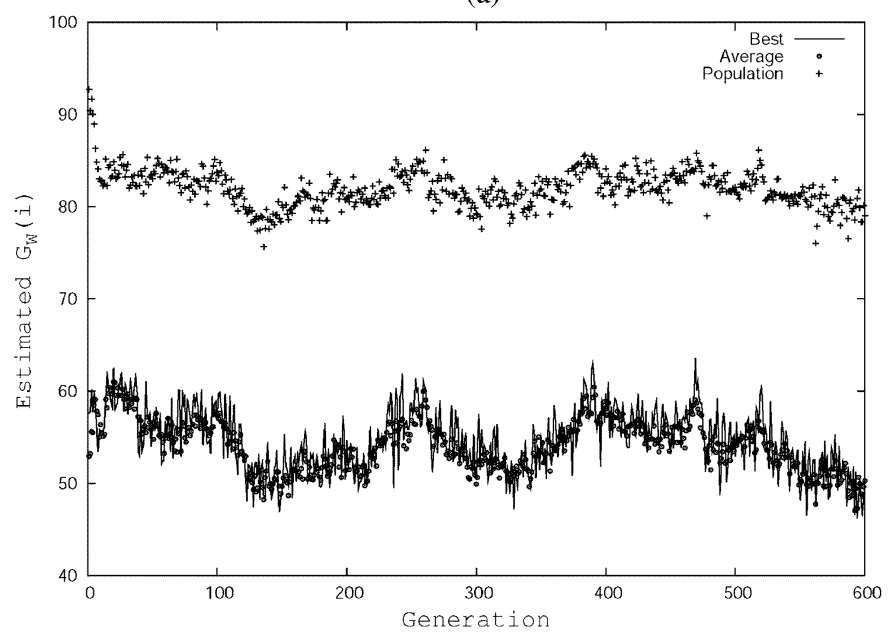

(b)

Fig. 6. Generalization performance given by $\operatorname{Best}\left(G_{\mathrm{SPOP}_{U}}\right)$, $\operatorname{Avg}\left(G_{\mathrm{SPOP}_{U}}\right)$, and $\operatorname{Pop}\left(G_{\mathrm{SPOP}_{U}}\right)$ measurements for coevolutionary learning systems with implicit fitness sharing across the generations for the four-choice IPD. The results of generalization performance during coevolutionary learning for (a) IFSD and (b) IFSR are shown. Each graph is obtained by averaging over 30 independent runs.

population of strategies from the point of view of generalization performance (as one observes from the local fluctuations in Fig. 6).

Similar observations are also made for PAR in Table III where the generalization performance of the population $\left(\operatorname{Pop}\left(G_{\text {SPOP }_{U}}\right)\right)$ is significantly higher compared to the generalization performance of individual evolved strategies $\left(\operatorname{Best}\left(G_{\text {SPOP }_{U}}\right)\right.$ and $\operatorname{Avg}\left(G_{\text {SPOP }_{U}}\right)$ ). These results for PAR are consistent with the emphasis of Pareto coevolution in selecting nondominated strategies in the coevolving population where each would maintain performance against different opponents that are increasingly higher in quality. The Pareto coevolution would result in the evolutionary search of strategies that have a greater degree of specialization, in which the combination of these individual strategies would result in higher generalization performance.

In addition, we have taken measurements of the generalization performance with respect to a biased sample $\hat{G}_{W}^{B}$ for various coevolutionary learning systems with implicit diversity maintenance and CCL without diversity maintenance. Table IV
TABLE IV

Summary of the RESUlts FOR THE GENERALIZATION PERFoRMANCE With RESPECT TO A BIASED SAMPLE GIVEN By Best $\left(G_{\mathrm{SPOP}_{U}}\right), \operatorname{Avg}\left(G_{\mathrm{SPOP}_{U}}\right)$, AND $\operatorname{Pop}\left(G_{\text {SPOP }_{U}}\right)$ FOR VARIOUS COEVOLUTIONARY LEARNING SYSTEMS With IMPLICIT DIVERSITY MAINTENANCE FOR THE FOUR-CHOICE IPD TAKen at the Final Generation. For Each System, the Average VALUE AND THE ERROR AT 95\% CONFIDENCE INTERVAL OVER 30 RUNS ARE Noted. Values in Bold Indicate That the Particular System HAS A SigNIFICANTLY HighER DiVERSITY LEVEL COMPARED TO THAT OF CCL-PM05 BASED ON THE PAIRED WILCOXON SIGNED RANK TEST FOR StATISTICAL SigNIFICANCE DifFERENCE AT THE 0.05 LEVEL OF SigNIFICANCE

\begin{tabular}{|c||c|c|c|}
\hline Experiment & $\operatorname{Best}\left(G_{\mathrm{SPOP}_{U}}\right)$ & $\operatorname{Avg}\left(G_{\mathrm{SPOP}_{U}}\right)$ & $\operatorname{Pop}\left(G_{\mathrm{SPOP}_{U}}\right)$ \\
\hline CCL-PM05 & $31.37 \pm 10.15$ & $29.37 \pm 8.13$ & $40.17 \pm 10.19$ \\
CFSD & $\mathbf{8 9 . 5 0} \pm \mathbf{6 . 4 7}$ & $\mathbf{9 1 . 4 3} \pm \mathbf{3 . 5 8}$ & $\mathbf{1 0 0 . 0 0} \pm \mathbf{0 . 0 0}$ \\
CFSR & $\mathbf{8 7 . 1 7} \pm \mathbf{8 . 2 9}$ & $\mathbf{8 0 . 7 7} \pm \mathbf{6 . 1 4}$ & $\mathbf{9 8 . 0 0} \pm \mathbf{3 . 0 5}$ \\
IFSD & $22.50 \pm 9.06$ & $16.20 \pm 4.10$ & $50.00 \pm 9.38$ \\
IFSR & $19.83 \pm 10.22$ & $20.00 \pm 8.18$ & $43.17 \pm 10.97$ \\
PAR & $36.00 \pm 14.15$ & $33.80 \pm 10.88$ & $\mathbf{6 3 . 8 3} \pm \mathbf{1 2 . 9 6}$ \\
RV & $12.83 \pm 6.10$ & $15.67 \pm 6.17$ & $26.33 \pm 10.35$ \\
\hline
\end{tabular}

summarizes the results of the $\operatorname{Best}\left(G_{\mathrm{SPOP}_{U}}\right), \operatorname{Avg}\left(G_{\mathrm{SPOP}_{U}}\right)$, and $\operatorname{Pop}\left(G_{\mathrm{SPOP}_{U}}\right)$ measurements for $\hat{G}_{W}^{B}$. In general, observations made from comparisons between coevolutionary learning systems with and without implicit diversity maintenance for $\hat{G}_{W}^{B}$ are similar to observations made for the case of $\hat{G}_{W}$.

For example, both CFSD and CFSR resulted in statistically significant and higher generalization performance for all measurements compared to that of CCL-PM05, while all measurements of generalization performance for $\mathrm{RV}$ are lower compared to CCL-PM05's (Table IV). The same observation is made for PAR where the $\operatorname{Pop}\left(G_{\mathrm{SPOP}_{U}}\right)$ measurement for $\hat{G}_{W}^{B}$ is statistically significantly higher compared to that of CCL-PM05. The only exception is coevolutionary learning with implicit fitness sharing (IFSD and IFSR) where the $\operatorname{Pop}\left(G_{\mathrm{SPOP}_{U}}\right)$ measurements for $\hat{G}_{W}^{B}$ are no longer statistically significantly different even though the values are still higher compared to those of CCL-PM05.

In addition, it is noted that although the relative generalization performance of different coevolutionary learning systems with implicit diversity maintenance remains unchanged (i.e., they can be ranked in the ascending order of RV, IFSR, IFSD, PAR, CFSR, and CFSD), the overall generalization performance measurements are lower with respect to a biased sample of "good" test strategies compared to the measurements made with respect to an unbiased sample of test strategies. These results are consistent with our earlier conclusion in [30] where it is more difficult for evolved strategies to perform well against strong game playing test strategies obtained from the multiple partial enumerative search.

\section{B. Generalization Performance of Coevolutionary Learning With Explicit Diversity Maintenance}

Table V summarizes the results of generalization performance measurements for $\hat{G}_{W}$ that have been made for different coevolutionary learning systems with explicit diversity maintenance in comparison to that of CCL-PM05. In general, results show that the application of explicit diversity maintenance (by increasing the $p_{m}$ setting) does not lead to an increase in the generalization performance of the top performing individual evolved strategies. Table $\mathrm{V}$ shows that $\operatorname{Best}\left(G_{\mathrm{SPOP}_{U}}\right)$ and 
TABLE V

SUMMARY OF THE RESUlTS FOR THE GENERALIZATION PERFORMANCE With RESPECT TO AN UNBIASED SAMPLE Given By Best $\left(G_{\text {SPOP }_{U}}\right)$, $\operatorname{Avg}\left(G_{\text {SPOP }_{U}}\right)$, AND Pop $\left(G_{\text {SPOP }_{U}}\right)$ FOR VARIOUS COEVOLUTIONARY LEARNING SYSTEMS WITH EXPLICIT DIVERSITY MAINTENANCE FOR THE FOUR-CHOICE IPD TAKEN AT THE FINAL GENERATION. For EACH SYSTEM, THE AVERAGE VALUE AND THE ERROR AT 95\% CONFIDENCE INTERVAL OVER 30 Runs ARE Noted. VALUES IN BOLd INDICATE THAT THE PARTICULAR SySTEM HaS A SignifiCANTLY Higher DiVERSITY LEVEl COMPARED TO THAT OF CCL-PM05 BASED ON THE PAIRED WILCOXON SigNED RANK TEST FOR StATISTICAL SigNifiCANCE DifFERENCE AT THE 0.05 LEVEL OF SigNIFICANCE

\begin{tabular}{|l|c|c|c|}
\hline Experiment & $\operatorname{Best}\left(G_{\mathrm{SPOP}_{U}}\right)$ & $\operatorname{Avg}\left(G_{\mathrm{SPOP}_{U}}\right)$ & $\operatorname{Pop}\left(G_{\mathrm{SPOP}_{U}}\right)$ \\
\hline CCL-PM05 & $61.56 \pm 5.91$ & $61.17 \pm 5.00$ & $73.67 \pm 4.56$ \\
CCL-PM10 & $54.15 \pm 6.37$ & $55.74 \pm 5.62$ & $77.84 \pm 4.14$ \\
CCL-PM15 & $51.41 \pm 6.68$ & $51.17 \pm 5.81$ & $75.09 \pm 5.14$ \\
CCL-PM20 & $55.04 \pm 5.90$ & $53.63 \pm 5.33$ & $\mathbf{8 2 . 6 1} \pm \mathbf{3 . 1 3}$ \\
CCL-PM25 & $46.78 \pm 6.45$ & $45.81 \pm 4.07$ & $\mathbf{8 1 . 9 3} \pm \mathbf{2 . 4 5}$ \\
\hline
\end{tabular}

$\operatorname{Avg}\left(G_{\mathrm{SPOP}_{U}}\right)$ measurements for $\hat{G}_{W}$ actually decrease. However, the generalization performance given by the population as a whole increases significantly if $p_{m}$ is sufficiently high. Table V shows that the $\operatorname{Pop}\left(G_{\mathrm{SPOP}_{U}}\right)$ measurements for $\hat{G}_{W}$ in the case of CCL-PM20 and CCL-PM25 are statistically significantly higher compared to the case of CCL-PM05.

The results show that increasing the level of variation in the population does not lead to the coevolutionary learning of individual strategies with higher generalization performance. However, the generalization performance of the population as a whole is increased. This observation suggests that the population is effectively speciating, with the individual specialist strategies outperforming smaller and different groups of opponents. The speciated population of coevolved strategies can be combined using a gating mechanism that effectively outperforms a larger number of opponents. Fig. 7 illustrates this observation where the large differences between the generalization performance for individual evolved strategies $\left(\operatorname{Best}\left(G_{\mathrm{SPOP}_{U}}\right)\right.$ and $\operatorname{Avg}\left(G_{\mathrm{SPOP}_{U}}\right)$ ) and the population $\left(\operatorname{Pop}\left(G_{\mathrm{SPOP}_{U}}\right)\right)$ are maintained on average throughout coevolution when $p_{m}$ is significantly increased (CCL-PM25). The same observation has been made earlier for IFSD and IFSR that also speciate the population (Fig. 6).

Table VI summarizes results for the generalization performance measurements with respect to a biased sample $\hat{G}_{W}^{B}$ for various coevolutionary learning systems with explicit diversity maintenance. We observe that the application of explicit diversity maintenance to coevolutionary learning by increasing the $p_{m}$ setting can have a positive impact in increasing the generalization performance $\hat{G}_{W}^{B}$ of the population as a whole. However, the increase is not statistically significant. This is not surprising as the evolved strategies are competing against a biased sample of strong game playing test strategies obtained from the multiple partial enumerative search.

\section{Impact of Diversity Maintenance on Diversity Levels in Coevolutionary Learning}

Results from the experiments show that not all applications of diversity maintenance techniques lead to positive and significant improvements on the generalization performance of coevolutionary learning. However, before any conclusion can be drawn, measurements of diversity levels in the population

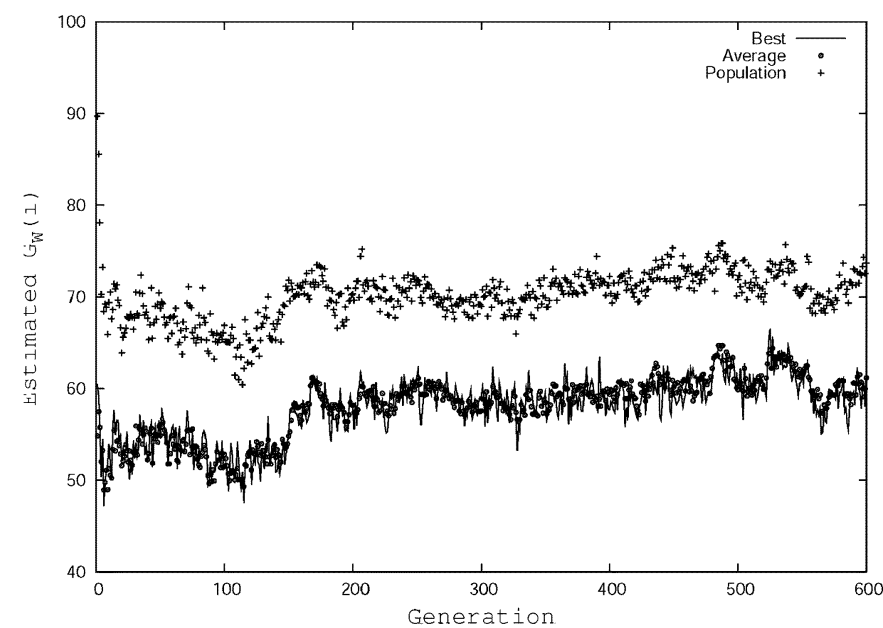

(a)

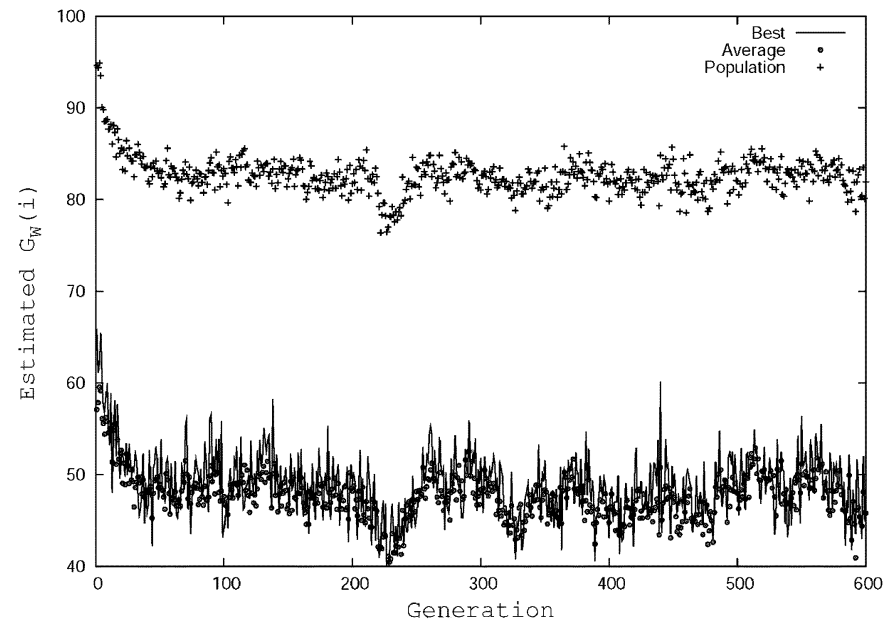

(b)

Fig. 7. Comparison of generalization performance given by $\operatorname{Best}\left(G_{\mathrm{SPOP}_{U}}\right)$, $\operatorname{Avg}\left(G_{\mathrm{SPOP}_{U}}\right)$, and $\operatorname{Pop}\left(G_{\mathrm{SPOP}_{U}}\right)$ measurements for coevolutionary learning systems with and without explicit diversity maintenance across the generations for the four-choice IPD. The results of generalization performance during coevolutionary learning for (a) CCL-PM05 and (b) CCL-PM25 are shown. Each graph is obtained by averaging over 30 independent runs.

TABLE VI

SUMMARY OF THE RESULTS FOR THE GENERALIZATION PERFORMANCE With RESPECT TO A BIASED SAMPLE GIVEN by $\operatorname{Best}\left(G_{\mathrm{SPOP}_{U}}\right), \operatorname{Avg}\left(G_{\mathrm{SPOP}_{U}}\right)$, AND $\operatorname{Pop}\left(G_{\mathrm{SPOP}_{U}}\right)$ FOR VARIOUS COEVOLUTIONARY LEARNING SYSTEMS

WITH EXPLICIT DIVERSITY MAINTENANCE FOR THE FOUR-CHOICE IPD

TAKen at the Final Generation. For EaCh System, THE AVERAGE VALUE AND THE ERROR AT 95\% CONFIDENCE INTERVAL OVER 30 RUNS ARE Noted. VAlues IN Bold INDICATE That the PARTICULAR SysteM HAS A SigNificANTLY HighER DIVERSITY LEVEL COMPARED TO THAT OF CCL-PM05 BASEd ON THE PAIRED WILCOXON SIGNED RANK TEST FOR

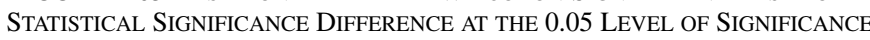

\begin{tabular}{|c|c|c|c|}
\hline Experiment & $\operatorname{Best}\left(G_{\text {SPOP }_{U}}\right)$ & $\operatorname{Avg}\left(G_{\mathrm{SPOP}_{U}}\right)$ & $\operatorname{Pop}\left(G_{\mathrm{SPOP}_{U}}\right)$ \\
\hline CCL-PM05 & $31.37 \pm 10.15$ & $29.37 \pm 8.13$ & $40.17 \pm 10.19$ \\
CCL-PM10 & $16.17 \pm 7.03$ & $18.23 \pm 5.83$ & $33.33 \pm 8.32$ \\
CCL-PM15 & $18.67 \pm 8.02$ & $17.83 \pm 6.48$ & $33.33 \pm 9.20$ \\
CCL-PM20 & $21.83 \pm 8.13$ & $24.93 \pm 5.39$ & $48.67 \pm 7.90$ \\
CCL-PM25 & $17.67 \pm 6.12$ & $17.97 \pm 3.88$ & $49.00 \pm 8.07$ \\
\hline
\end{tabular}

for different coevolutionary learning systems with and without diversity maintenance would need to be performed. Table VII summarizes the results of genotypic and phenotypic diversity 
TABLE VII

SUMMARY OF GENOTYPIC AND PHENOTYPIC DIVERSITIES FOR VARIOUS COEVOLUTIONARY LEARNING SYSTEMS WITH DIVERSITY MAINTENANCE FOR THE FOUR-CHOICE IPD TAKEN AT THE FINAL GENERATION. FOR EACH SYSTEM, THE AVERAGE VALUE AND THE ERROR AT 95\% CONFIDENCE INTERVAL OVER 30 RuNS ARE NOTED. VALUES IN BOLD INDICATE THAT THE PARTICULAR SYSTEM HAS A SigNIFICANTLY HighER DiVERSITY LEVEL COMPARED TO THAT OF CCL-PM05 BASED ON THE PAIRED WILCOXON Signed Rank Test For Statistical Significance DifFERENCE AT THE 0.05 LEVEL OF SigNificanCE

\begin{tabular}{|c||c||c||}
\hline Experiment & Genotypic Diversity & Phenotypic Diversity \\
\hline CCL-PM05 & $0.18 \pm 0.02$ & $1.23 \pm 0.27$ \\
CCL-PM10 & $\mathbf{0 . 3 3} \pm \mathbf{0 . 0 3}$ & $\mathbf{2 . 1 2} \pm \mathbf{0 . 3 4}$ \\
CCL-PM15 & $\mathbf{0 . 4 1} \pm \mathbf{0 . 0 2}$ & $\mathbf{2 . 8 5} \pm \mathbf{0 . 1 7}$ \\
CCL-PM20 & $\mathbf{0 . 5 0} \pm \mathbf{0 . 0 2}$ & $\mathbf{2 . 9 9} \pm \mathbf{0 . 1 9}$ \\
CCL-PM25 & $\mathbf{0 . 5 9} \pm \mathbf{0 . 0 2}$ & $\mathbf{3 . 3 4} \pm \mathbf{0 . 0 2}$ \\
CFSD & $\mathbf{0 . 3 1} \pm \mathbf{0 . 0 2}$ & $0.83 \pm 0.21$ \\
CFSR & $\mathbf{0 . 3 6} \pm \mathbf{0 . 0 3}$ & $1.20 \pm 0.27$ \\
IFSD & $\mathbf{0 . 5 5} \pm \mathbf{0 . 0 3}$ & $\mathbf{2 . 8 2} \pm \mathbf{0 . 1 0}$ \\
IFSR & $\mathbf{0 . 3 7} \pm \mathbf{0 . 0 3}$ & $\mathbf{3 . 1 5} \pm \mathbf{0 . 0 7}$ \\
PAR & $\mathbf{0 . 3 9} \pm \mathbf{0 . 0 4}$ & $\mathbf{2 . 4 2} \pm \mathbf{0 . 2 4}$ \\
RV & $0.23 \pm 0.03$ & $\mathbf{1 . 7 2} \pm \mathbf{0 . 2 5}$ \\
\hline
\end{tabular}

measurements for different coevolutionary learning systems that are taken at the final generation. The results provide a means to determine the impact of diversity maintenance on the diversity levels in the population of coevolutionary learning through the comparison of diversity levels between coevolutionary learning systems with diversity maintenance and CCL system (CCL-PM05) that is not designed specifically to introduce and maintain high diversity levels.

In general, the results on the measurements of diversity levels are consistent and in agreement with the design motivations of the respective coevolutionary learning systems with diversity maintenance. For coevolutionary learning with explicit diversity maintenance, it is expected that the introduction of more variations to the evolved strategies in the population by increasing the $p_{m}$ setting would lead to an increase in the genotypic diversity levels. Table VII shows that the increase in genotypic diversity levels is statistically significant (e.g., comparing the genotypic diversity level of CCL-PM25 with that of CCL-PM05). Furthermore, the statistically significant increase that is shown in Table VII in phenotypic diversity levels is consistent with the use of the direct lookup table strategy representation. More genotypic variation in the population of strategies would lead to more variation in the behavioral interactions, which are reflected by the higher phenotypic diversity levels.

For coevolutionary learning systems with implicit fitness sharing, the general increase in diversity levels (genotypic and phenotypic) is consistent with the original design motivation of speciating the population by creating niches of evolved strategies having different and unique behaviors [29], [44]. Table VII shows that both genotypic and phenotypic diversity levels for IFSD and IFSR are statistically significantly higher compared to that of CCL-PM05. A similar observation can also be made for the case of PAR in comparison with CCL-PM05. The higher diversity levels would be a result of the evolution and subsequent maintenance of a large set of mutually nondominating strategies (e.g., the entire population) [33].

For coevolutionary learning with RV, Table VII shows that a higher phenotypic diversity level is introduced and maintained in the population compared to that of CCL-PM05. However, RV has only a slightly higher genotypic diversity level in the population compared to that of CCL-PM05. Despite this, the results are consistent with the design motivation of the technique. In particular, the technique does not seek to diversify a population into niches, but instead, aims to encourage the population to engage in interactions [25]. This would result in the coevolution of a population of strategies responding differently to one another, leading to an increase in fitness variations that would be observed as an increase in the phenotypic diversity level.

The only exception is coevolutionary learning with competitive fitness sharing. In our case studies, the application of competitive fitness sharing does not lead to an increase in the phenotypic diversity level of the population in coevolutionary learning. Table VII shows that the phenotypic diversity levels for CFSD and CFSR are lower compared to that of CCL-PM05. This observation is different compared to the observation that has been reported earlier in [27]. In particular, the study uses a diversity measurement that counts the number of unique strategy responses above a significant usage, which is similar to the phenotypic diversity measurement. This discrepancy may be a result of applying coevolutionary learning to different games.

Despite this, the use of competitive fitness sharing does lead to a positive and significant increase in the genotypic diversity level in the population that is shown in Table VII. These results are verified through a closer inspection of evolved strategies. It is observed that evolved strategies in the population have engaged mostly with "full defection," which would result in similar average payoffs that lead to low phenotypic diversity. However, observations on the representation of these evolved strategies show differences on the parts that are not accessed during game play interactions, which nevertheless would result in higher genotypic diversity.

In comparing the relative levels of diversity that are introduced and maintained due to applications of different diversity maintenance techniques in coevolutionary learning, both implicit fitness sharing and explicit diversity maintenance appear to be particularly effective. Fig. 8 plots the average genotypic and phenotypic diversity levels of different coevolutionary learning systems across the generations. Both figures indicate that the implicit fitness sharing and explicit diversity maintenance (at sufficiently high $p_{m}$ settings) can introduce and maintain higher diversity levels (genotypic and phenotypic) in the population throughout the evolutionary process compared to other diversity maintenance techniques.

Thus far, we have presented and discussed separately the impact of different diversity maintenance techniques in introducing and maintaining different diversity levels (genotypic and phenotypic) in coevolutionary learning. Here, we investigate whether there are relationships between genotypic and phenotypic diversity in coevolutionary learning with diversity maintenance. Our analysis would be based on a series of scatter plots (which have been employed previously in [34] for analysis) of genotypic and phenotypic diversity. Each point in the 


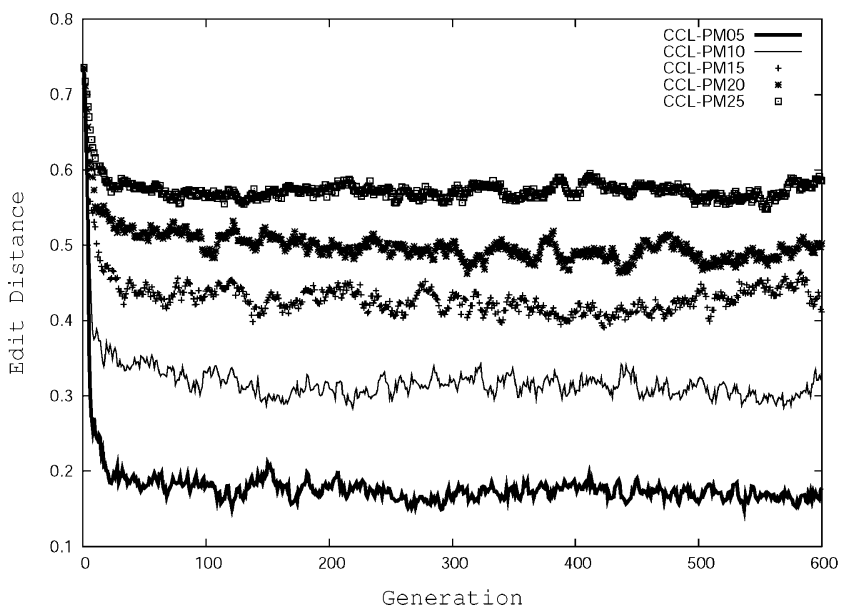

(a)

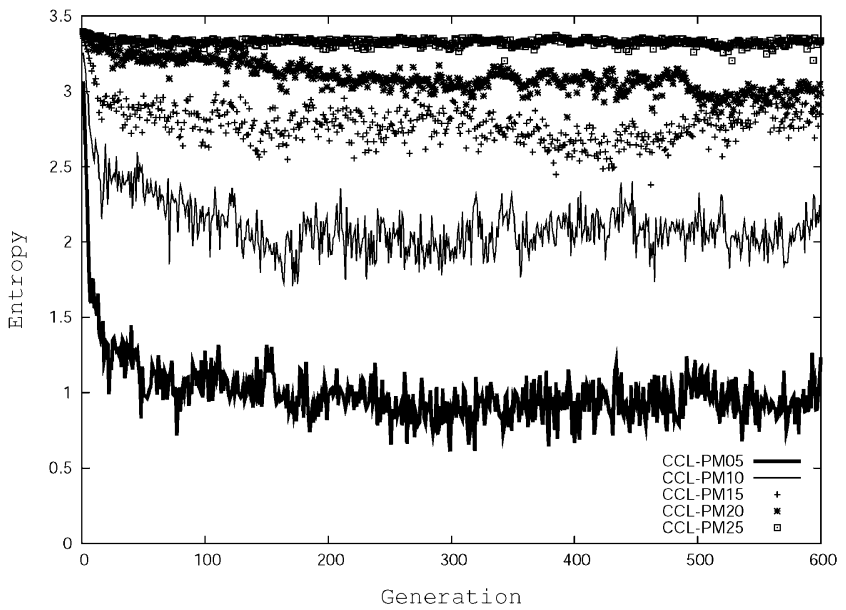

(c)

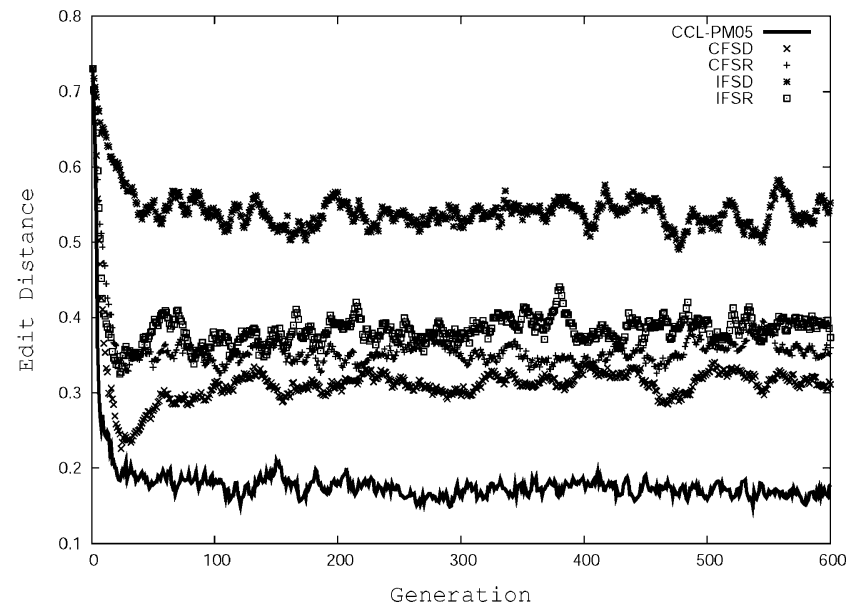

(b)

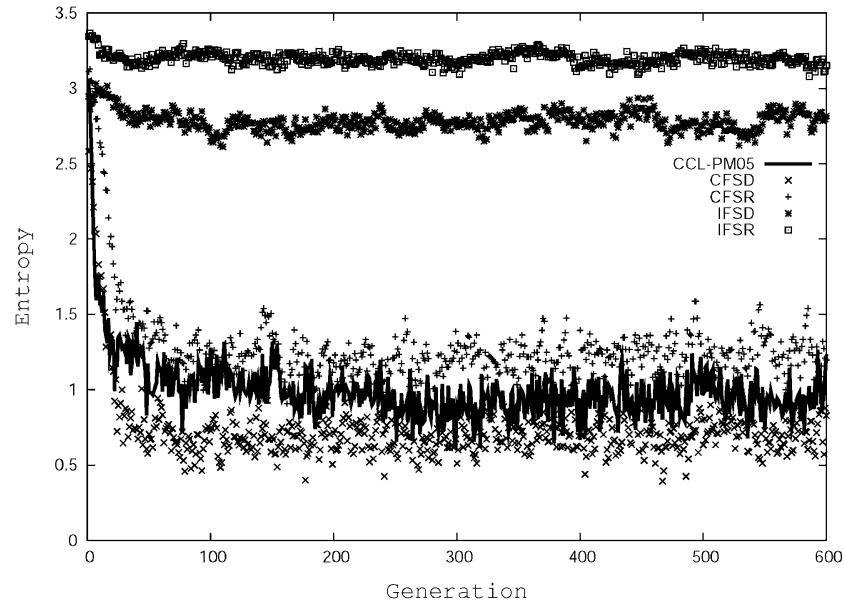

(d)

Fig. 8. Comparison of genotypic and phenotypic diversity for various coevolutionary learning systems across the generations for the four-choice IPD. (a) and (b) Comparison of the genotypic diversity for the case of CCL-PM05 with that of coevolutionary learning with explicit diversity maintenance (CCL-PM10, CCLPM15, CCL-PM20, and CCL-PM25) and implicit diversity maintenance (CFSD, CFSR, IFSD, and IFSR), respectively. (c) and (d) Comparison of the phenotypic diversity for the case of CCL-PM05 with that of coevolutionary learning with explicit diversity maintenance (CCL-PM10, CCL-PM15, CCL-PM20, and CCLPM25) and implicit diversity maintenance (CFSD, CFSR, IFSD, and IFSR), respectively. Each graph is obtained by averaging over 30 independent runs.

scatter plot represents one population in a particular generation from each of the 30 independent runs. Since each coevolutionary learning system is run for 600 generations and repeated 30 times, the scatter plot would have a total of $600 \times 30$ points. The scatter plot may provide information on the interactions and possible relationships between genotypic and phenotypic diversity as a result of the application of specific diversity maintenance techniques in coevolutionary learning.

We have compared the genotypic-phenotypic diversity scatter plots for coevolutionary learning with and without explicit diversity maintenance. Fig. 9 illustrates two general observations that we have made. First, there is a trend of higher genotypic diversity occurring with higher phenotypic diversity for CCL-PMs at higher $p_{m}$ settings (such as CCL-PM20). The higher $p_{m}$ settings would introduce more genotypic variations, which in turn, would lead to more variations in the behavioral interactions (phenotypic diversity) in coevolutionary learning. Second, CCL-PMs with higher $p_{m}$ settings can better maintain both genotypic and phenotypic diversity, e.g., the points are distributed more towards the top-right region of the scatter plot for CCL-PM20 compared to that of CCL-PM05.
We have also compared the genotypic-phenotypic diversity scatter plots for various coevolutionary learning systems with implicit diversity maintenance. In general, we observe a trend of higher genotypic diversity occurring with higher phenotypic diversity for coevolutionary learning with implicit fitness sharing (Fig. 10) and also Pareto coevolution. The observation for such a trend is less apparent for the case of coevolutionary learning with RV and more so with competitive fitness sharing (Fig. 10). For our case studies, the positive relationship of genotypic and phenotypic diversity can be explained by considering the effect of speciation in the population to niches of evolved strategies having different and unique behaviors. A speciated population would have high genotypic and phenotypic diversity. The speciation effect would be very pronounced in IFSD.

\section{Relationship Between Generalization Performance and Diversity in Coevolutionary Learning}

The results of generalization performance and diversity measurements from the case studies show that the introduction and maintenance of diversity through the applications of diversity 


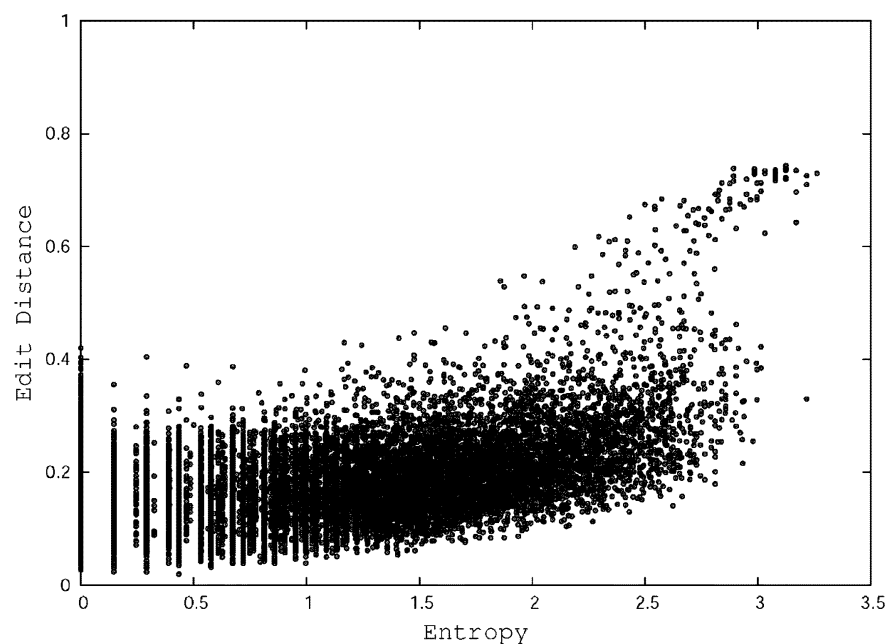

(a)

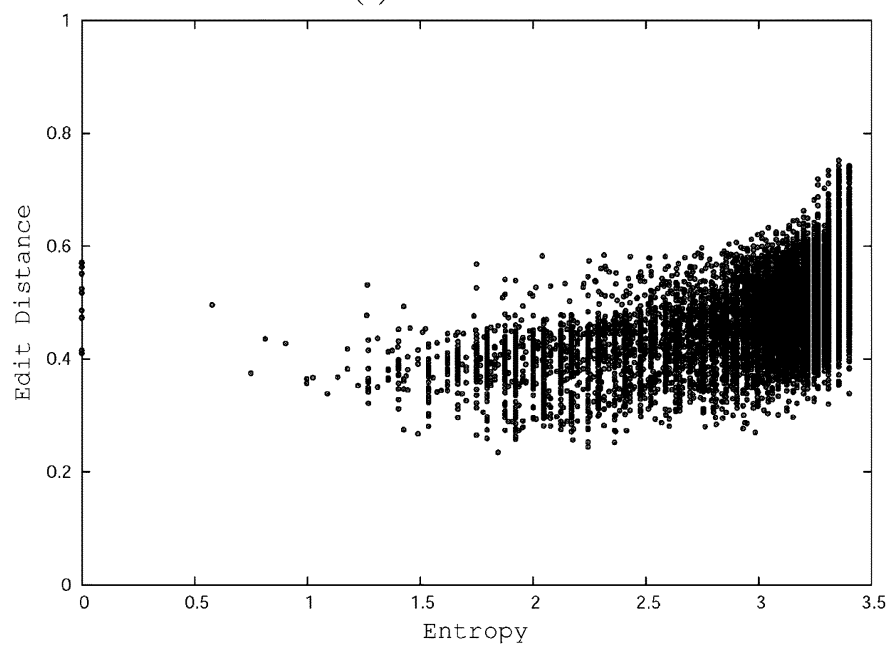

(b)

Fig. 9. Comparison of genotypic-phenotypic diversity scatter plots for coevolutionary learning systems with explicit diversity maintenance for the four-choice IPD. Each figure plots the population's genotypic diversity against the population's phenotypic diversity. Each point represents one population in a particular generation from each of the 30 independent runs. There are altogether $600 \times 30$ points. (a) CCL-PM05. (b) CCL-PM20.

maintenance techniques do not necessarily lead to improvements on the generalization performance of coevolutionary learning. A case in point is the use of coevolutionary learning with RV where the resulting generalization performance is lower compared to CCL without diversity maintenance, regardless of whether the generalization performance measurements are made with respect to individual evolved strategies or with respect to the population.

However, it is not necessarily true that a diversity maintenance technique that does not introduce and maintain high diversity levels during coevolution will lead to lower generalization performance. A case in point is the use of the competitive fitness sharing in coevolutionary learning. Results have shown that the application of competitive fitness sharing significantly improves on the generalization performance in all measurements, i.e., regardless of whether an unbiased or biased sample of test strategies is used, or whether the measurements are made on individual evolved strategies or the population as a whole. This

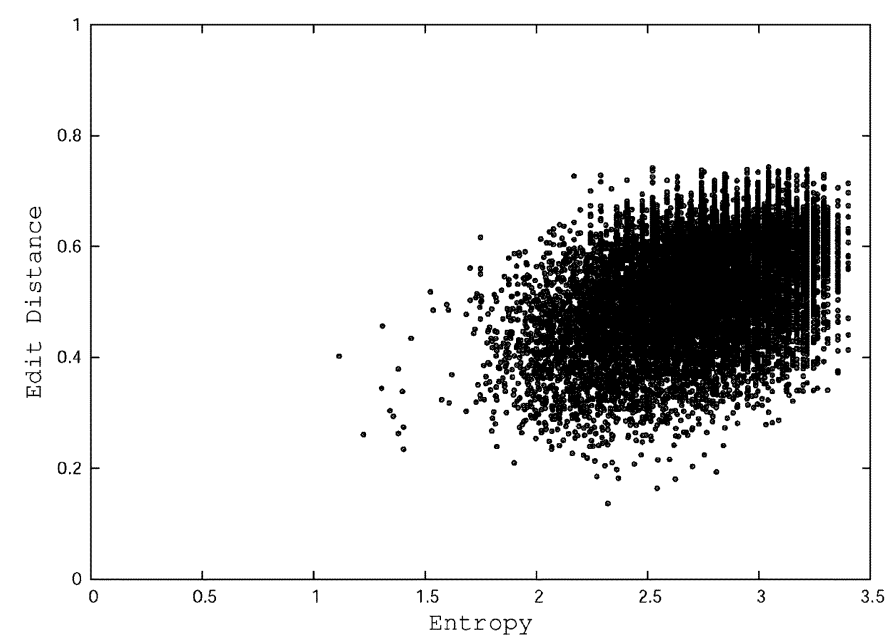

(a)

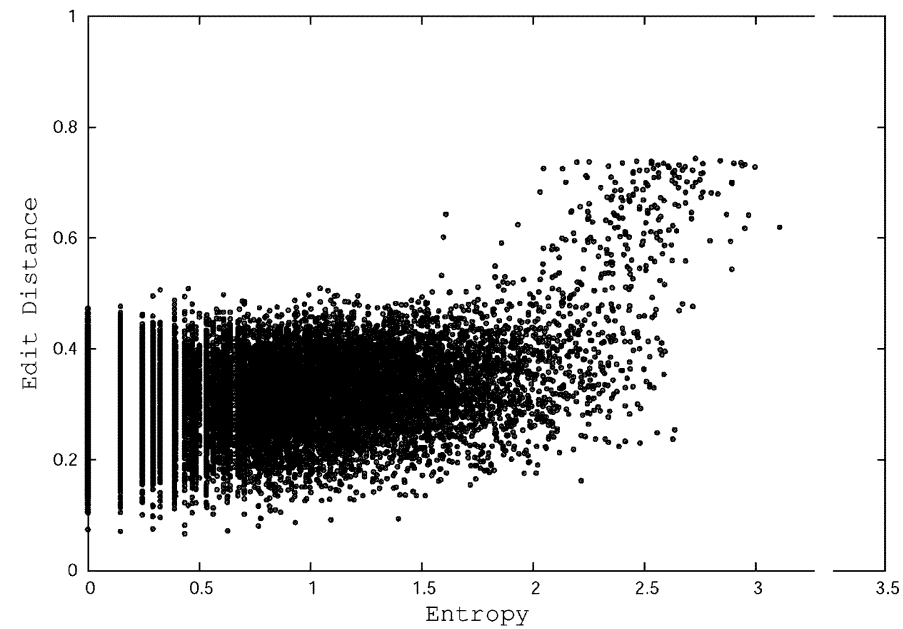

(b)

Fig. 10. Comparison of genotypic-phenotypic diversity scatter plots for coevolutionary learning systems with implicit diversity maintenance for the fourchoice IPD. Each figure plots the population's genotypic diversity against the population's phenotypic diversity. Each point represents one population in a particular generation from each of the 30 independent runs. There are altogether $600 \times 30$ points. (a) IFSD. (b) CFSD.

is despite coevolutionary learning systems with competitive fitness sharing not having higher diversity levels compared to systems using other diversity maintenance techniques.

The success of competitive fitness sharing in improving the generalization performance of coevolutionary learning appears to depend on how the strategy fitness is determined using (1). The emphasis of the selection process on strategies outperforming opponents that few others could leads to the coevolutionary learning of strategies that play "full defection" more often. Given that an "all defect" strategy would obtain the maximum possible generalization performance (defined by the win-lose outcome), a coevolutionary learning system that evolves a population of strategies effectively playing "all defect" would have the highest generalization performance. In this case, it would appear that diversity plays little role in improving the generalization performance of coevolutionary learning [we note the similarity in the genotypic-phenotypic diversity scatter plots for CFSD in Fig. 10(b) and for CCL-PM05 in Fig. 9(a)]. 


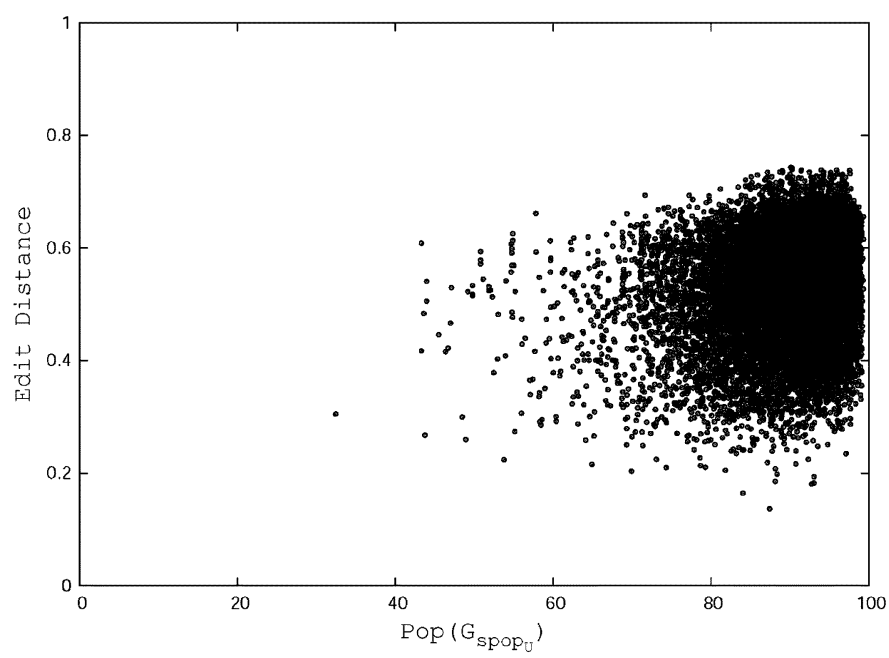

(a)

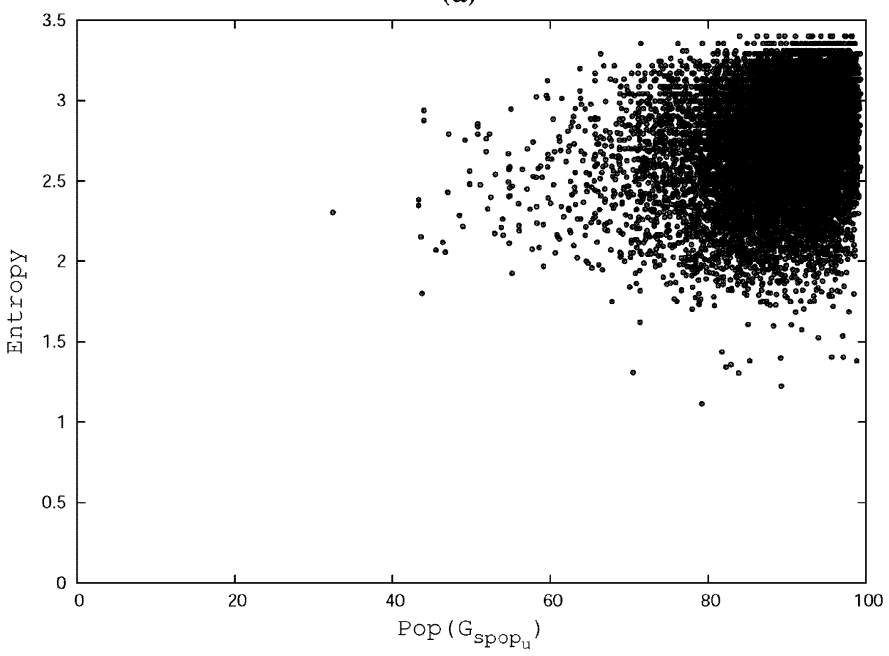

(b)

Fig. 11. Comparison of diversity-generalization scatter plots for coevolutionary learning systems with implicit fitness sharing for the four-choice IPD. Each figure plots the population's diversity against the population's generalization performance estimated by $\operatorname{Pop}\left(G_{\mathrm{SPOP}_{U}}\right)$. Each point represents one population in a particular generation from each of the 30 independent runs. There are altogether $600 \times 30$ points. (a) IFSD. (b) IFSD.

However, one general observation that we have made consistently from the case studies is that the introduction and maintenance of diversity that lead to the speciation of the population to unique niches of specialized strategies have a positive and significant impact on the generalization performance of coevolutionary learning if the population of strategies can be combined (e.g., using a gating mechanism as in [29]). We have conducted an analysis based on diversity-generalization scatter plots to investigate this issue. Fig. 11 shows that the speciation effect of a coevolving population is particularly effective in improving the generalization performance through the population of speciated strategies obtained from the application of implicit fitness sharing. One can observe a trend of high diversity (genotypic and phenotypic) occurring with high generalization performance of the population for coevolutionary learning with implicit fitness sharing. Similar observations can be made to some extent for Pareto coevolution and coevolutionary learning with explicit diversity maintenance, although it is apparent that implicit fitness sharing has a more pronounced speciation effect to coevolutionary learning that can improve generalization performance.

Although overall our case studies have not been able to establish a clear positive relationship between the generalization performance (as we define it) and diversity in coevolutionary learning, there are some diversity maintenance techniques, e.g., implicit fitness sharing, that seem to be able to boost the generalization performance when the population of coevolved strategies can be combined. Some past studies such as [29] and [71] have also suggested that diversity in the population can be exploited in coevolutionary learning to improve "out-of-sample" performance. In those approaches, diversity plays an important role in coevolutionary learning through speciation to obtain a diverse set of solutions (each solution specializing with respect to different groups of test cases) that as a whole has the potential for higher performance. However, the issues of quantification of population diversity and performance measurement remain open research questions.

\section{CONCLUSION}

In this paper, we have conducted a detailed empirical study to investigate the issue of whether there is a relationship between generalization performance and diversity in coevolutionary learning. Understanding this issue is important given that the lack of diversity in the population may have a negative impact on the generalization performance of coevolutionary learning. More importantly, specific conditions may be identified with potential implications for design choices in coevolutionary search algorithms whereby diversity can be exploited to improve the generalization performance in coevolutionary learning.

We have used games as they provide a natural framework to study coevolutionary learning. The interactions between the coevolving solutions in the population can be framed as game playing. This allows one to study the two main properties of a coevolutionary learning system, i.e., decision making and learning, in detail. With games, one is interested in the ability of coevolutionary learning system to search for strategies that can outperform a large number of different opponents. We have conducted case studies involving IPD games to systematically investigate the impact of various diversity maintenance techniques on the generalization performance of coevolutionary learning. The case studies compare coevolutionary learning with and without diversity maintenance and address two shortcomings in previous studies through a series of quantitative measurements for both the generalization performance and diversity levels in the population of various coevolutionary learning systems.

We have been motivated to answer two related questions in our studies: 1) what is the right form of diversity that would be beneficial for generalization, and 2) what amount of the "right" form of diversity is needed for good generalization? Here, results from our case studies have shown that the introduction and maintenance of diversity in the population of coevolutionary learning do not necessarily lead to a significant increase in the generalization performance. Although some diversity maintenance techniques have been shown to significantly improve the 
generalization performance of coevolutionary learning, there are cases (e.g., RV) where a positive relationship between the generalization performance and diversity has not been observed and established in our experiments.

We have also investigated the coverage of the whole strategy space by the population of coevolved strategies. In particular, we have generated a large number of test strategies and for each test strategy, we have checked whether the coevolved population contains a strategy capable of winning against that test strategy. Lower values of this population measure would signify that the coevolved population is "homogeneous" and there is a large pool of possible test strategies for which coevolutionary learning has not evolved effective strategies to compete against. On the other hand, larger values of this population measure mean a good coverage of the strategy space by the coevolved population. We have observed that diversity maintenance that led to a speciated population (e.g., implicit fitness sharing) did increase the coverage of the strategy space. For other diversity maintenance techniques, we have not observed a strong correlation between increased diversity and strategy space coverage.

However, the population coverage measurement using an ideal gating mechanism only indicates the potential of the coevolved population to do well against arbitrary test strategies. Given a test strategy, we would need a mechanism for choosing the "right" coevolved strategy from the population. Ensemble approaches can be used to combine different expertise in a diverse population together to form a stronger strategy [29], [71]. This is a direction for our future research. An ensemble approach can be regarded as an automatic divide-and-conquer approach to complex problem solving. Different individuals in the ensemble will be specialists in dealing with different aspects of the entire problem. As a result, the ensemble as a whole will be a stronger solution.

The study has considered different forms of fitness measurements (e.g., implicit diversity maintenance techniques) in coevolutionary learning that are evaluated using a generalization performance measure based on the win-lose function. However, the motivation of our study is not constrained to the use of a specific choice of fitness measure in coevolutionary learning and its evaluation by a specific generalization performance measure. We use the generalization performance measure based on the win-lose function to simplify the analysis of the experiments since we know that "all defect" is the strategy with the maximum generalization performance. Other functions such as average payoffs can also be used although the analysis will be much more difficult since we may not know a priori the strategy having the maximum generalization performance. Further studies on this topic will be of interest although intuitively we would expect that some forms of diversity in coevolutionary learning can be exploited to evolve a population of strategies that can be combined to gain a higher generalization performance. It is very difficult to evolve a single strategy that performs best against a large number of different opponents for any generalization performance measure. We also note that although the results might be sensitive to some parameter settings (e.g., population size, strategy representation, and others), we speculate that the results are qualitatively robust in that a diverse population has the potential for higher generalization performance.

The empirical study here is a first step towards a deeper understanding of the relationship between the generalization performance and diversity in coevolutionary learning. In the context of the IPD games, other frameworks for quantitative analysis involving nonlocal adaptation to compare performance between populations and fingerprints to measure diversity in the population can be employed to investigate further how diversity can be related to generalization performance in coevolutionary learning. Future studies may also focus on addressing how diversity can be exploited for problem contexts other than games that allows the design of algorithmic implementations of coevolutionary learning that can lead to improvements in generalization performance.

\section{ACKNOWLEDGMENT}

The authors would like to thank the anonymous associate editor and reviewers for their comments that have helped to improve this paper. They would also like to thank The Centre of Excellence for Research in Computational Intelligence and Applications (CERCIA), School of Computer Science, The University of Birmingham, U.K., for providing computing support in running the experiments.

\section{REFERENCES}

[1] X. Yao, "Evolutionary artificial neural networks," Proc. IEEE, vol. 87, no. 9, pp. 1423-1447, Sep. 1999.

[2] X. Yao and Y. Xu, "Recent advances in evolutionary computation," J. Comput. Sci. Technol., vol. 21, no. 1, pp. 1-18, 2006.

[3] X. Yao, "Introduction," Informatica, vol. 18, Special Issue on Evolutionary Computation, pp. 375-376, 1994.

[4] D. B. Fogel, Evolutionary Computation: Toward a New Philosophy of Machine Intelligence. Piscataway, NJ: IEEE Press, 1995.

[5] T. Bäck, U. Hammel, and H. P. Schwefel, "Evolutionary computation: Comments on the history and current state," IEEE Trans. Evol. Comput., vol. 1, no. 1, pp. 3-17, Apr. 1997.

[6] D. B. Fogel, "An introduction to simulated evolutionary optimization," IEEE Trans. Neural Netw., vol. 5, no. 1, pp. 3-14, Jan. 1994.

[7] C. A. C. Coello, "A comprehensive survey of evolutionary-based multiobjective optimization techniques," Knowl. Inf. Syst., vol. 1, no. 3, pp. 129-156, 1999.

[8] Y. Liu, X. Yao, and T. Higuchi, "Evolutionary ensembles with negative correlation learning," IEEE Trans. Evol. Comput., vol. 4, no. 4, pp. 380-387, Nov. 2000.

[9] N. García-Pedrajas, C. Hervás-Martnez, and D. Ortiz-Boyer, "Cooperative coevolution of artificial neural network ensembles for pattern classification," IEEE Trans. Evol. Comput., vol. 9, no. 3, pp. 271-302, Jun. 2005.

[10] D. B. Fogel, A. D. Blair, and R. Mikkulainen, "Special issue: Evolutionary computation and games," IEEE Trans. Evol. Comput., vol. 9, no. 6, pp. 537-539, Dec. 2005.

[11] K. Chellapilla and D. B. Fogel, "Evolution, neural networks, games, and intelligence," Proc. IEEE, vol. 87, no. 9, pp. 1471-1496, Sep. 1999.

[12] H. Dawid, H. L. Poutré, and X. Yao, "Computational intelligence in economic games and policy design," IEEE Comput. Intell. Mag., vol. 3 , no. 4 , pp. 22-26, Nov. 2008.

[13] R. Axelrod, "The evolution of strategies in the iterated prisoner's dilemma," in Genetic Algorithms and Simulated Annealing, L. D. Davis, Ed. New York: Morgan Kaufmann, 1987, ch. 3, pp. 32-41.

[14] S. Luke and R. P. Wiegand, A. Barry, Ed., "When coevolutionary algorithms exhibit evolutionary dynamics," in Proc. Workshop Understanding Coevol., Theory Anal. Coevol. Algorithms, 2002, pp. 236-241.

[15] E. Popovici and K. D. Jong, "Relationships between internal and external metrics in co-evolution," in Proc. IEEE Congr. Evol. Comput., Edinburgh, U.K., Sep. 25, 2005, pp. 2800-2807.

[16] D. Cliff and G. F. Miller, "Coevolution of pursuit and evasion ii: Simulation methods and results," in Proc. 4th Int. Conf. Simul. Adapt. Behav. (From Animals to Animats), 1996, vol. 4, pp. 506-515. 
[17] S. Nolfi and D. Floreano, "Co-evolving predator and prey robots: Do "arms races" arise in artificial evolution?," Artif. Life, vol. 4, no. 4, pp. 311-335, 1998.

[18] S. G. Ficici and J. B. Pollack, "Challenges in coevolutionary learning: Arms-race dynamics, open-endedness, and mediocre stable states," in Proc. 6th Int. Conf. Artif. Life, C. Adami, R. K. Belew, H. Kitano, and C. Taylor, Eds., 1998, pp. 238-247.

[19] R. A. Watson and J. B. Pollack, "Coevolutionary dynamics in a minimal substrate," in Proc. Genetic Evol. Comput. Conf., 2001, pp. 702-709.

[20] S. Y. Chong, M. K. Tan, and J. D. White, "Observing the evolution of neural networks learning to play the game of Othello," IEEE Trans. Evol. Comput., vol. 9, no. 3, pp. 240-251, Jun. 2005.

[21] J. Noble, "Finding robust Texas Hold'em poker strategies using Pareto coevolution and deterministic crowding," in Proc. Int. Conf. Mach. Learn. Appl., 2002, pp. 233-239.

[22] K. O. Stanley and R. Miikkulainen, "Competitive coevolution through evolutionary complexification," J. Artif. Intell. Res., vol. 21, pp. 63-100, 2004.

[23] T. P. Runarsson and S. M. Lucas, "Coevolution versus self-play temporal difference learning for acquiring position evaluation in smallboard go," IEEE Trans. Evol. Comput., vol. 9, no. 6, pp. 540-551, Dec. 2005.

[24] E. D. de Jong and J. B. Pollack, "Ideal evaluation from coevolution," Evol. Comput., vol. 12, no. 2, pp. 159-192, 2004.

[25] J. Cartlidge and S. Bullock, "Combating coevolutionary disengagement by reducing parasite virulence," Evol. Comput., vol. 12, no. 2, pp. 193-222, 2004.

[26] P. J. Darwen and X. Yao, "Co-evolution in iterated prisoner's dilemma with intermediate levels of cooperation: Application to missile defense," Int. J. Comput. Intell. Appl., vol. 2, no. 1, pp. 83-107, 2002.

[27] C. D. Rosin and R. K. Belew, "New methods for competitive coevolution," Evol. Comput., vol. 5, no. 1, pp. 1-29, 1997.

[28] S. G. Ficici, "Solution concepts in coevolutionary algorithms," Ph.D. dissertation, Dept. Comput. Sci., Brandeis Univ., Boston, MA, 2004.

[29] P. J. Darwen and X. Yao, "Speciation as automatic categorical modularization," IEEE Trans. Evol. Comput., vol. 1, no. 2, pp. 101-108, Jul. 1997.

[30] S. Y. Chong, P. Tiňo, and X. Yao, "Measuring generalization performance in co-evolutionary learning," IEEE Trans. Evol. Comput., vol. 12, no. 4, pp. 479-505, Aug. 2008.

[31] P. J. Darwen and X. Yao, "On evolving robust strategies for iterated prisoner's dilemma," in Progress in Evolutionary Computation, ser. Lecture Notes in Artificial Intelligence. Berlin, Germany: SpringerVerlag, 1995, vol. 956, pp. 276-292.

[32] S. G. Ficici and J. B. Pollack, "Pareto optimality in coevolutionary learning," in Lecture Notes in Computer Science. Berlin, Germany: Springer-Verlag, 2001, vol. 2159, pp. 316-325.

[33] J. Noble and R. A. Watson, "Pareto coevolution: Using performance against coevolved opponents in a game as dimensions for Pareto selection," in Proc. Genetic Evol. Comput. Conf, 2001, pp. 493-500.

[34] E. K. Burke, S. Gustafson, and G. Kendall, "Diversity in genetic programming: An analysis of measures and correlation with fitness," IEEE Trans. Evol. Comput., vol. 8, no. 1, pp. 47-62, Feb. 2004.

[35] J. P. Rosca, "Entropy-driven adaptive representation," in Proc. Workshop Genetic Programming: From Theory to Real-World Appl., Tahoe City, CA, 1995, pp. 23-32.

[36] K. A. DeJong, "An analysis of the behavior of a class of genetic adaptive systems," Ph.D. dissertation, Dept. Comput. Commun. Sci., Univ. Michigan, Ann Arbor, MI, 1975.

[37] D. E. Goldberg and J. Richardson, "Genetic algorithms with sharing for multimodal function optimization," in Proc. 2nd Int. Conf. Genetic Algorithms Their Appl., 1987, pp. 41-49.

[38] K. Deb and D. E. Goldberg, "An investigation of niche and species formation in genetic function optimization," in Proc. 3rd Int. Conf. Genetic Algorithms, 1989, pp. 42-50.

[39] S. W. Mahfoud, "Niching methods for genetic algorithms," Ph.D. dissertation, Dept. General Eng., Univ. Illinois at Urbana-Champaign, Urbana, IL, 1995.

[40] P. J. Darwen and X. Yao, "Every niching method has its niches: Fitness sharing and implicit fitness sharing compared," in Lecture Notes in Computer Science. Berlin, Germany: Springer-Verlag, 1996, vol. 1141 , pp. 398-407.

[41] A. Pétrowski, "A new selection operator dedicated to speciation," in Proc. 7th Int. Conf. Genetic Algorithms, 1997, pp. 144-151.

[42] M. Bessaou, A. Pétrowski, and P. Siarry, "Island model cooperating with speciation for multimodal optimization," in Lecture Notes in Computer Science. Berlin, Germany: Springer-Verlag, 2000, vol. 1917, pp. 437-446.
[43] R. K. Ursem, "Diversity-guided evolutionary algorithms," in Lecture Notes in Computer Science. Berlin, Germany: Springer-Verlag, 2002, vol. 2439, pp. 462-474.

[44] P. J. Darwen, "Co-evolutionary learning by automatic modularization with speciation," Ph.D. dissertation, School Comput. Sci., Univ. New South Wales, Sydney, N.S.W., Australia, Nov. 1996.

[45] R. E. Smith, S. Forrest, and A. S. Perelson, "Searching for diverse, cooperative populations with genetic algorithms," Evol. Comput., vol. 1, no. 2, pp. 127-149, 1992.

[46] P. J. Darwen and X. Yao, "A dilemma for fitness sharing with a scaling function," in Proc. IEEE Conf. Evol. Comput., Perth, W.A., Australia, 1995, pp. 166-171.

[47] R. Axelrod, The Evolution of Cooperation. New York: Basic Books, 1984.

[48] R. Axelrod, "Effective choice in the prisoner's dilemma," J. Conflict Resolution, vol. 24, no. 1, pp. 3-25, Mar. 1980.

[49] R. Axelrod, "More effective choice in the prisoner's dilemma," J. Conflict Resolution, vol. 24, no. 3, pp. 379-403, Sept. 1980.

[50] S. Y. Chong and X. Yao, "Behavioral diversity, choices, and noise in the iterated prisoner's dilemma," IEEE Trans. Evol. Comput., vol. 9, no. 6, pp. 540-551, Dec. 2005.

[51] P. J. Darwen and X. Yao, "Why more choices cause less cooperation in iterated prisoner's dilemma," in Proc. IEEE Congr. Evol. Comput., Seoul, Korea, May 27-30, 2002, pp. 987-994.

[52] X. Yao and P. J. Darwen, "How important is your reputation in a multiagent environment," in Proc. IEEE Conf. Syst. Man Cybern., Tokyo, Japan, Oct. 12-15, 1999, pp. 575-580.

[53] P. J. Darwen and X. Yao, "Does extra genetic diversity maintain escalation in a co-evolutionary arms race," Int. J. Knowl.-Based Intell. Eng. Syst., vol. 4, no. 3, pp. 191-200, July 2000.

[54] R. Axelrod and W. D. Hamilton, "The evolution of cooperation," Science, vol. 211, pp. 1390-1396, 1981.

[55] D. B. Fogel, "The evolution of intelligent decision making in gaming," Int. J. Cybern. Syst., vol. 22, pp. 223-236, 1991.

[56] D. B. Fogel, "Evolving behaviors in the iterated prisoner's dilemma," Evol. Comput., vol. 1, no. 1, pp. 77-97, 1993.

[57] D. B. Fogel, "On the relationship between the duration of an encounter and the evolution of cooperation in the iterated prisoner's dilemma," Evol. Comput., vol. 3, no. 3, pp. 349-363, 1996.

[58] B. A. Julstrom, "Effects of contest length and noise on reciprocal altruism, cooperation, and payoffs in the iterated prisoner's dilemma," in Proc. 7th Int. Conf. Genetic Algorithms, San Francisco, CA, 1997, pp. 386-392.

[59] S. Y. Chong, J. Humble, G. Kendall, J. Li, and X. Yao, "Learning IPD strategies through co-evolution," in The Iterated Prisoners Dilemma: 20 Years On, G. Kendall, X. Yao, and S. Y. Chong, Eds. Singapore: World Scientific, 2007, ch. 3, pp. 63-87.

[60] X. Yao, Y. Liu, and P. J. Darwen, "How to make best use of evolutionary learning," in Complex Systems - From Local Interactions to Global Phenomena, R. Stocker, H. Jelinck, B. Burnota, and T. Bossomaier, Eds. Amsterdam, The Netherlands: IOS Press, 1996, pp. 229-242.

[61] D. Ashlock and B. Powers, "The effect of tag recognition on non-local adaptation," in Proc. Congr. Evol. Comput., 2004, pp. 2045-2051.

[62] P. G. Harrald and D. B. Fogel, "Evolving continuous behaviors in the iterated prisoner's dilemma," BioSystems, vol. 37, Special Issue on the Prisoners Dilemma, pp. 135-145, 1996.

[63] N. Franken and A. P. Engelbrecht, "Particle swarm optimization approaches to coevolve strategies for the iterated prisoner's dilemma," IEEE Trans. Evol. Comput., vol. 9, no. 6, pp. 562-579, Dec. 2005.

[64] B. V. Gnedenko and G. V. Gnedenko, Theory of Probability. New York: Taylor \& Francis, 1998.

[65] A. Ekárt and S. Németh, "A metric for genetic programs and fitness sharing," in Lecture Notes in Computer Science. Berlin, Germany: Springer-Verlag, 2000, vol. 1802, pp. 259-2701.

[66] E. D. de Jong, R. A. Watson, and J. B. Pollack, "Reducing bloat and promoting diversity using multi-objective methods," in Proc. Genetic Evol. Comput. Conf., 2001, pp. 11-18.

[67] U. M. O'Reilly, "Using a distance metric on genetic programs to understand genetic operators," in Proc. IEEE Int. Conf. Syst. Man Cybern., 1997 , pp. 4092-4097.

[68] A. Ekárt and S. Nemeth, "Maintaining the diversity of genetic programs," in Lecture Notes in Computer Science. Berlin, Germany: Springer-Verlag, 2002, vol. 2278, pp. 162-171.

[69] S. Gustafson, "An analysis of diversity in genetic programming," $\mathrm{Ph} . \mathrm{D}$. dissertation, School Comput. Sci., Univ. Nottingham, Nottingham, U.K., 2004.

[70] D. Ashlock and E.-Y. Kim, "Fingerprinting: Visualization and automatic analysis of prisoner's dilemma strategies," IEEE Trans. Evol. Comput., vol. 12, no. 5, pp. 647-659, Oct. 2008. 
[71] K.-J. Kim and S.-B. Cho, "Knowledge into evolutionary speciated checkers players," IEEE Trans. Evol. Comput., vol. 9, no. 6, pp. 615-627, Dec. 2005.

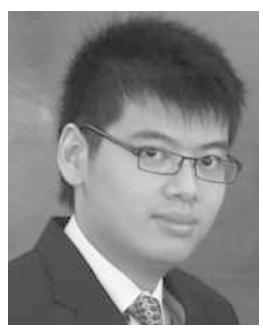

Siang Yew Chong (M'99) received the B.Eng. (honors) and M.Eng.Sc. degrees in electronics engineering from Multimedia University (MMU), Melaka, Malaysia, in 2002 and 2004, respectively, and the Ph.D. degree in computer science from the University of Birmingham, Birmingham, U.K., in 2007.

He was a Research Associate with the Centre of Excellence for Research in Computational Intelligence and Applications (CERCIA), School of Computer Science, University of Birmingham, in 2007. Currently, he is an Honorary Research Fellow with the School of Computer Science, University of Birmingham. He joined the School of Computer Science, University of Nottingham, Malaysia Campus (UNMC) in 2008 and is currently a member of the Automated Scheduling, Optimisation and Planning (ASAP) Research Group, School of Computer Science, University of Nottingham, U.K. He has coedited the book The Iterated Prisoners' Dilemma: 20 Years On (Singapore: World Scientific, 2007). His main research interests include evolutionary computation, machine learning, and game theory.

Dr. Chong was awarded the Outstanding Ph.D. Dissertation Award by the IEEE Computational Intelligence Society (CIS) in 2009 for his work on coevolutionary learning. He was awarded the Student Travel Grant for the 2003 Congress on Evolutionary Computation (CEC'03). He is an Associate Editor of the IEEE TRANSACTIONS ON COMPUTATIONAL INTELLIGENCE AND AI IN GAMES.

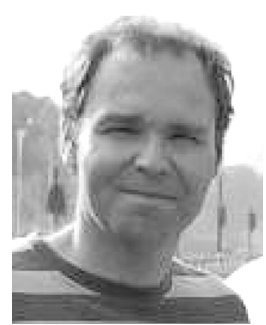

Peter Tiňo received the M.Sc. degree from the Slovak University of Technology, Bratislava, Slovakia, in 1988 and the Ph.D. degree from the Slovak Academy of Sciences, Bratislava, Slovakia, in 1997.

He was a Fullbright Fellow at the NEC Research Institute, Princeton, NJ, from 1994 to 1995 . He was a Postdoctoral Fellow at the Austrian Research Institute for AI, Vienna, Austria, from 1997 to 2000, and a Research Associate at the Aston University, U.K., from 2000 to 2003. Currently, he is with the School of Computer Science, University of Birmingham, Birmingham, U.K., where since 2003 he has been a Senior Lecturer. His main research interests include probabilistic modeling and visualization of structured data, statistical pattern recognition, dynamical systems, evolutionary computation, and fractal analysis.

Dr. Tiňo was awarded the Fullbright Fellowship in 1994 and the U.K.-Hong Kong Fellowship for Excellence in 2008. He was awarded the Outstanding Paper of the Year from the IEEE TRANSACTIONS ON NEURAL NETWORKS with T. Lin, B. G. Horne, and C. L. Giles in 1998 for the work on recurrent neural networks. He won the 2002 Best Paper Award at the International Conference on Artificial Neural Networks with B. Hammer. He is on the editorial board of several journals.

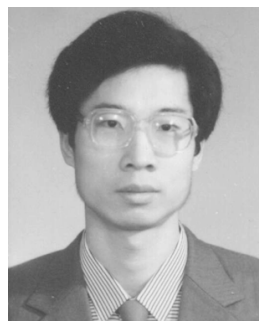

Xin Yao (M'91-SM'96-F'03) received the B.Sc. degree from the University of Science and Technology of China (USTC), Hefei, China, in 1982, the M.Sc. degree from the North China Institute of Computing Technology, Beijing, China, in 1985, and the Ph.D. degree from USTC in 1990.

He was an Associate Lecturer and Lecturer from 1985 to 1990 at USTC, while working towards his Ph.D. He took up a Postdoctoral Fellowship in the Computer Sciences Laboratory, Australian National University (ANU), Canberra, A.C.T., Australia, in 1990, and continued his work on simulated annealing and evolutionary algorithms. He joined the Knowledge-Based Systems Group, CSIRO Division of Building, Construction and Engineering, Melbourne, Vic., Australia, in 1991, working primarily on an industrial project on automatic inspection of sewage pipes. He returned to Canberra in 1992 to take up a lectureship at the School of Computer Science, University College, University of New South Wales (UNSW), Australian Defence Force Academy (ADFA), where he was later promoted to a Senior Lecturer and Associate Professor. He moved to the University of Birmingham, U.K., as a Professor of Computer Science in 1999. Currently, he is the Director of the Centre of Excellence for Research in Computational Intelligence and Applications (CERCIA), University of Birmingham, Birmingham, U.K., a Distinguished Visiting Professor of the USTC, and a visiting professor of three other universities. His major research interests include evolutionary computation, neural network ensembles, and real-world applications. He has more than 300 refereed publications in these areas.

Dr. Yao was awarded the President's Award for Outstanding Thesis by the Chinese Academy of Sciences for his Ph.D. work on simulated annealing and evolutionary algorithms. He won the 2001 IEEE Donald G. Fink Prize Paper Award for his work on evolutionary artificial neural networks. He was the Editor-in-Chief (2003-08) of the IEEE TRANSACTIONS ON EVOLUTIONARY COMPUTATION. He is an Associate Editor or editorial board member of 12 international journals. He is the Editor of the World Scientific Book Series on Advances in Natural Computation. He has given more than 50 invited keynote and plenary speeches at international conferences worldwide. 